\title{
A NEW APPROACH TO THE CONSTRUCTION OF CONFORMAL FIELD THEORIES*
}

\author{
M. Caselle \\ Istituto Nazionale di Fisica Nucleare \\ 10125 Torino, Italy \\ K. S. Narain \\ International Center for Theoretical Physics \\ 34014 Trieste, Italy \\ and \\ Theory Division, CERN, Geneva
}

\begin{abstract}
We propose a generalization of Coulomb gas approach to Rational conformal field theories to higher number of bosons. This is achieved by letting the screening charges to live on rational lattices and not just on lattices associated with Lie groups. The characters are constructed using a certain "Weyl" group associated with these rational lattices and they form finite dimensional representations of the modular group. We further propose a method to construct correlation functions. We study in particular few examples for 2-boson case and obtain a bosonic realization of the spin- $4 / 3$ parafermionic series. Several non-trivial checks are also made in order to show that our prescription for computing correlation functions indeed gives correct results.
\end{abstract}

DFTT 23/88

December 1988

* Work supported in part by Ministero della Pubblica Istruzione. 
The study of 2-dimensional conformal invariant field theories (CFT), while important from the statistical mechanics point of view [1] has also been playing a central role in the context of string theories. By taking suitable combinations of CFT's one can construct classical solutions of the string theory $[2,3]$. In particular a study of rational CFT's (RCFT) (i.e. having finite number of primary fields associated to some chiral algebra) is a first step in the classification of all CFT's. A considerable amount of work has been done in this direction [4-8]. Large numbers of RCFT's are known to date and there are different ways to construct them. In this paper we shall generalize Dotsenko Fateev Coulomb gas approach $[9,10]$ to more than one scalars. In fact this generalization of Dotsenko-Fateev procedure has already been done, in a limited sense, by Fateev and Zamolodchikov [11] and by Fateev and Lykyanov [12] to obtain RCFT's associated to chiral algebras including higher spin fields. These algebras are realized explicitly in terms of free scalars. The screening operators are constructed as vertex operators associated with the root lattice of SU(N) Lie algebras, which commute with the chiral algebra and as a result one could construct infinite numbers of null states starting from a given primary field.

In the following we shall proceed in the opposite direction. We shall specify the screening operators associated with an arbitrary lattice, not just with some group lattice. The basic assumption we make is on the existence of a chiral algebra which would commute with these screening operators. Associated with these screening operators one defines a "Weyl" group which acts on certain conjugacy classes of this lattice. This way one can construct characters that form finite dimensional representations of the modular group. A subset of them, obeying positivity conditions on the characters and other basic requirements such as the existence of a translationally invariant ground state are candidates for RCFT's . Using these characters we further propose a prescription to construct correlation functions on the torus whose degeneration limit gives correlation function on the sphere and therefore to obtain the operator algebra for these models. Although our procedure of constructing characters is inspired by the Feigin-Fuchs construction, one needs more complex tools to describe the null state structure of the models. We believe that our procedure to construct RCFT's exhausts all those RCFT's whose characters can be described as lattice sums.

We analyze the case of 2-bosons systems and obtain several series of RCFT's. In particular we can realize the spin $4 / 3$ parafermionic series in terms of 2 bosons. We also obtain the $\frac{S U^{k}(2) \times S U^{1}(2) \times S U^{1}(2)}{S U^{k+2}(2)}$ series as well as some other isolated cases. We study the correlation functions for some of these models on the torus and on the sphere. Our results on the sphere coincide with the known results and thereby provide a nontrivial check of the entire procedure. In the examples that we studied for the 2-bosons case the unitary models that we obtain always happen to be in one of the GKO [13] coset series. However since our approach is completely different from the GKO construction it could provide a useful tool to investigate whether GKO cosets exhaust all the unitary RCFT's. In any case realization of some of these models in term of bosons is useful as it is a much simpler description, it enables one to construct correlation functions on the torus and it is a natural starting point to search for new bosonic chiral algebras.

In the next section we outline the general procedure and derive conditions on the lattice defining the screening operators. We discuss the procedure to construct the characters and obtain conditions to get modular invariant partition functions. We also discuss the 
prescriptions to compute correlation functions for the models on the torus and on the sphere. In section 3 and 4 we discuss examples with 2 scalars, obtain the corresponding characters (sect.3) and show that in the known cases they coincide with the known characters, we also compute correlation functions (sect.4) for some of these models using the prescription outlined in sect. 2 and show that they agree with the known results. The last section is devoted to some concluding remark. 


\section{Section 2: General Procedure}

\section{A] CHARACTERS}

We start with a number (say d) of free scalars $\phi_{i},(\mathrm{i}=1,2, \ldots \mathrm{d})$. We shall use the vectorial notation and denote these scalar fields as $\vec{\phi}$ (a d-dimensional vector). Define the stress energy tensor as:

$$
T_{z z}=\frac{1}{4} \partial_{z} \vec{\phi} \partial_{z} \vec{\phi}+i \vec{\alpha}_{0} \partial_{z}^{2} \vec{\phi}
$$

where $\vec{\alpha}_{0}$ is a fixed vector and represents the charge at infinity. $T_{\bar{z} \bar{z}}$ is defined analogously. Using this $T_{z z}$ we can obtain the dimensions of vertex operators. Thus if:

$$
V_{\beta}(z)=: e^{i \vec{\beta} \vec{\phi}(z)}:
$$

then it carries the dimensions $(h, \bar{h})$ given by:

$$
h=\vec{\beta}^{2}-2 \vec{\alpha}_{0} \vec{\beta}=\bar{h}
$$

One can compute the central charge associated with the Virasoro algebra in the usual way:

$$
c=d-24 \vec{\alpha}_{0}^{2}
$$

Next step is to define the screening operators. Screening operators must necessarily carry dimension 1, as all the singular terms in the operator product of the screening operators with $\mathrm{T}$ must be a total derivative. This means that if a screening operator is

$$
V_{\vec{e}}=: e^{i \vec{e} \vec{\phi}}:
$$

then $\vec{e}$ must satisfy:

$$
\vec{e}^{2}-2 \vec{\alpha}_{0} \vec{e}=1
$$

Thus $\vec{e}$ must lie on the sphere defined by the above equation. Let us pick $2 \mathrm{~d}$ such vectors $\vec{e}_{a}, \mathrm{a}=1, . .2 \mathrm{~d}$. We assume that there exist a chiral algebra, which must have $\mathrm{d}$ independent generators, one of them being $\mathrm{T}$, such that the singular terms in the operator product of the screening operators $\left\{\vec{e}_{a}\right\}$ with the chiral algebra is a total derivative.

The primary fields in the theory are all the vertex operators $V_{\vec{\beta}}$ for arbitrary $\vec{\beta}$. We are interested in constructing a completely degenerate representation of this chiral algebra in order to obtain RCFT. This means that we must keep only those $\vec{\beta}$ such that the chiral moduli constructed from $V_{\vec{\beta}}$ contains infinite numbers of null states. We can construct infinite number of null states using the screening operators. Indeed

$$
\prod_{i=1}^{n} \oint d z_{i} V_{\vec{e}_{a}}\left(z_{i}\right) V_{\vec{\beta}-n \vec{e}_{a}}(0)
$$


would be a null state in the module $\left\{V_{\vec{\beta}}\right\}$, provided the contour of $z_{i}$ can be closed, due to the fact that the singular terms in the operator product of $V_{\vec{e}_{a}}$ with the chiral algebra is a total derivative. One can show that such a closed contour exists and gives a non vanishing contribution provided:

$$
\vec{\beta} \vec{e}_{a}=\frac{1+n_{a}}{2} \vec{e}_{a}^{2}-\frac{1+m_{a}}{2}
$$

for some positive integers $n_{a}$ and $m_{a}$ (for $m_{a}=0$ the integral gives the same state as $\left.V_{\vec{\beta}}(0)\right)$. The resulting null state is at level $n_{a} m_{a}$ above $V_{\vec{\beta}}(0)$. In fact all the chiral quantum numbers of the resulting null state are the same as that of $V_{\vec{\beta}-n_{a} \vec{e}_{a}}(0)$ (no sum w.r.t. a). Since we want to construct a completely degenerate representation of the chiral algebra, we will require $\vec{\beta}$ to satisfy eq. (2.3) for each $\vec{e}_{a}$. Let us denote $\Gamma$ the set of all $\vec{\beta}$ 's that satisfy eq. (2.3) for all a. Our RCFT must contain the identity sector. Thus the zero vector must belong to $\Gamma$. This implies using eq (2.3) that $\vec{e}_{a}^{2}=$ rational number for all a. Let $k_{a}$ be the smallest positive integer such that $k_{a} \vec{e}_{a}^{2}=$ integer, then eq (2.3) has the symmetry

$$
\left(n_{a}, m_{a}\right) \rightarrow\left(n_{a}+r k_{a}, m_{a}+r k_{a} \vec{e}_{a}^{2}\right)
$$

for any integer $\mathrm{r}$. Using this symmetry we can see that $\Gamma$ forms a lattice. Indeed eq (2.3) is linear so if $\vec{\beta}$ and $\vec{\beta}^{\prime}$ satisfy (2.3) then $r \vec{\beta}+s \vec{\beta}^{\prime}$ also satisfies (2.3) for any integers $\mathrm{r}$ and $\mathrm{s}$, by if necessary using the symmetry $(2.4)$ in order to satisfy the positivity condition on $\left(n_{a}, m_{a}\right)$.

The $S L(2 C)$ invariant ground state must have a null vector at level 1 . This can be seen by noticing that $V_{2 \vec{\alpha}_{0}}(0)$ is also a state with weight 0 . Thus we can identify $V_{0}(0)$ with $V_{2 \vec{\alpha}_{0}}(0)$. Now if we substitute $\vec{\beta}=2 \vec{\alpha}_{0}$ in eq (2.3) then for each a, $n_{a}=m_{a}=1$ is a solution. This means that in fact there are several null states at level 1 and they are given by $V_{\vec{e}_{a}}(0)$ for each $\vec{e}_{a}$. Now in order to get a completely degenerate representation $V_{\vec{e}_{a}}(0)$ must also give rise to infinite number of null states. Thus $\vec{e}_{a}$ must belong to $\Gamma$. Using eq. (2.3) this imply that

$$
2 k_{a} \vec{e}_{a} \vec{e}_{b} \in \mathbf{Z} \quad \forall a, b
$$

Consider a lattice $\Gamma^{\prime}$ such that

$$
\Gamma^{\prime}=\left\{\vec{\alpha} \mid \exists n_{a} \in \mathbf{Z}, \vec{\alpha}=\sum_{a} n_{a} k_{a} \vec{e}_{a}\right\}
$$

then eqs. (2.5) and (2.6) imply that $\sqrt{2} \Gamma^{\prime}$ is an even, integral lattice. Now eq. (2.5) implies that $\sqrt{2} \Gamma \subset\left(\sqrt{2} \Gamma^{\prime}\right)^{*}$. On the other hand for any $\beta \in \frac{1}{2}\left(\Gamma^{\prime}\right)^{*}$ we can always find positive $\left(n_{a}, m_{a}\right)$ satisfying $(2.3)$ by if necessary using the symmetry $(2.4)$. Thus $\Gamma^{\prime}=\frac{1}{2} \Gamma^{*}$. Since $\sqrt{2} \Gamma^{\prime}$ is integral, $\sqrt{2} \Gamma^{\prime} \subset\left(\sqrt{2} \Gamma^{\prime}\right)^{*}=\sqrt{2} \Gamma$ thus we can form conjugacy classes $\left(\frac{\Gamma}{\Gamma^{\prime}}\right)$. These classes and the lattice $\Gamma^{\prime}$ will play an important role in the construction of characters in the following.

Another ingredient we will need to construct characters is the action of a discrete group $\mathrm{G}$ (analog of the Weyl group) on the conjugacy classes $\left(\frac{\Gamma}{\Gamma^{\prime}}\right)$. Let $\vec{\beta} \in \Gamma$, thus there exist 
$\left(n_{a}, m_{a}\right)$ which satisfy $(2.3)$ We define an element $g_{a} \in G$ such that

$$
g_{a} \vec{\beta}=\vec{\beta}+n_{a} \vec{e}_{a}
$$

Note that $g_{a}$ is not well defined on $\Gamma$. Indeed using the symmetry $(2.4):\left(n_{a}, m_{a}\right) \rightarrow$ $\left(n_{a}+r_{a} k_{a}, m_{a}+r_{a} k_{a} \vec{e}_{a}^{2}\right)$

$$
g_{a} \vec{\beta}=\vec{\beta}+n_{a} \vec{e}_{a}+r_{a} k_{a} \vec{e}_{a}=\vec{\beta}+n_{a} \vec{e}_{a}+\vec{\beta}^{\prime}
$$

where $\vec{\beta}^{\prime} \in \Gamma^{\prime}$. Thus the action of $g_{a}$ is defined only modulo $\Gamma^{\prime}$. In fact it is easy to see that

$$
g_{a}:\left(\frac{\Gamma}{\Gamma^{\prime}}\right) \rightarrow\left(\frac{\Gamma}{\Gamma^{\prime}}\right)
$$

and the map is one-to-one and onto. Furthermore $g_{a}^{2}=1$. We define $\mathrm{G}$ as the group generated by all the $g_{a}$ 's , Let $\mathrm{N}$ be the order of $\left(\frac{\Gamma}{\Gamma^{\prime}}\right)$ then it is clear that $g_{a} \in P_{N}$ where $P_{N}$ is the permutation group of $\mathrm{N}$ elements. Thus $G \subset P_{N}$ and hence $\mathrm{G}$ is a finite group. Note that $g_{a}$ 's in fact give the first level null states for a given $\vec{\beta}$, and they will therefore play a crucial role in the construction of characters. There are some additional symmetries in our system that preserve the dimensions of the operators. For example an obvious symmetry is $\vec{\beta} \rightarrow 2 \vec{\alpha}_{0}-\vec{\beta}$ ( or $\vec{\beta} \rightarrow 2 \vec{\alpha}_{0}-\vec{\beta}^{*}$ where $\left.\vec{\beta}^{*}=\frac{2 \vec{\alpha}_{0}\left(\vec{\beta} \alpha_{0}\right)}{\alpha_{0}^{2}}-\vec{\beta}\right)$. Sometimes there can be some extra symmetries. Let $G_{0}$ be the group of such symmetries:

$$
G_{0} \ni g: \vec{\beta} \rightarrow \vec{\alpha}_{0}+g\left(\vec{\beta}-\vec{\alpha}_{0}\right)
$$

where $g \in O(d)$.

In particular let $\vec{\beta}=0$ (i.e. the ground state). In general there can be many vectors (besides the trivial one: $\vec{\beta}=2 \vec{\alpha}_{0}$ ) which also carry zero dimension. Let $\beta_{i}$ be such a vector. Then there exist $g_{i} \in O(d)$ such that $\vec{\beta}_{i}=\vec{\alpha}_{0}-g_{i} \vec{\alpha}_{i}$. We assume that:

i] $g_{i}$ is an automorphism of the entire lattice $\Gamma$, as well as $\Gamma^{\prime}$. Thus $g_{i}$ induce an action on $\left(\frac{\Gamma}{\Gamma^{\prime}}\right)$ which is again one-to-one and onto and is an element of $P_{N}$.

ii] The group $G_{0}$ generated by all such $g_{i}$ 's is an automorphism of $\Gamma$ and $\Gamma^{\prime}$.

Both $G_{0}$ and $G$ acting on $\left(\frac{\Gamma}{\Gamma^{\prime}}\right)$ are subgroups of $P_{N}$. We can form a bigger group $\tilde{G} \subset P_{N}$, which contains $G_{0}$ and $G$ and is generated by $g_{a}$ and $g_{i}$, then we require that:

iii] $G_{0}$ is a normal subgroup of $\tilde{G}$

The generic case is $G_{0}=(1,-1)$ which certainly satisfies the above two conditions. But there are also special cases, as we shall see in some examples, where the group is larger.

Finally we also need to define a $\mathbf{Z}_{2}$ grading in $\tilde{G}$. Anticipating the form of the characters and the fact that $g_{a}$ gives the first level null states which have to be subtracted, we define 
$g_{a}$ as an odd element for each a. On the other hand since $g_{i}$ is dimension preserving and essentially plays the role of identifying different states (such as $\vec{\beta}$ with $2 \vec{\alpha}_{0}-\vec{\beta}^{*}$ ) we define $g_{i}$ as even elements. As $\tilde{G}$ is generated by $g_{i}$ and $g_{a}$ we can extend this grading to $\tilde{G}$ provided there are no obstructions. We assume that:

iv] the grading $c\left(g_{i}\right)=-1$ and $c\left(g_{a}\right)=+1$ can be extended to $\tilde{G}$.

In those cases where (iv) is not true, we will see that all the characters are identically zero and therefore we must discard such models. However we will see that already for $d=2$ there are many examples that satisfy the above requirements.

We want to form characters, using the above lattice, that form a finite dimensional representation of the modular group. We define for each element $[\beta] \in \frac{\Gamma}{\Gamma^{\prime}}$ where $[\beta]$ denotes the class of $\beta$ (in the following, being understood that $\beta \in \Gamma$ we shall omit the vectorial notation:

$$
\chi_{[\beta]}=\sum_{g \in \tilde{G}} c(g) L(g \beta)
$$

where

$$
L(g \beta)=\frac{1}{\eta^{d}} \sum_{p \in \Gamma^{\prime}} q^{\left(g \beta-\alpha_{0}+p\right)^{2}}
$$

we now show that $\chi_{[\beta]}$ 's form a finite dimensional representation of the modular group. By a Poisson resummation:

$$
L(g \beta, \tau) \rightarrow \frac{1}{\eta\left(-\frac{1}{\tau}\right)^{d}} \sum_{p \in\left(\Gamma^{\prime}\right)^{*}} e^{-\frac{2 \pi i}{\tau} \frac{p^{2}}{4}+2 \pi i p\left(g \beta-\alpha_{0}\right)}
$$

writing $k=\frac{1}{2} p \in \frac{1}{2}\left(\Gamma^{\prime}\right)^{*}=\Gamma$ and expressing $k=g \beta^{\prime}+p^{\prime}-\alpha_{0}$ where $p^{\prime} \in \Gamma^{\prime}$,

$$
\begin{gathered}
L(g \beta, \tau) \rightarrow \frac{1}{\eta\left(\tau^{\prime}\right)^{d}} \sum_{\beta^{\prime} \in\left(\frac{\Gamma}{\Gamma^{\prime}}\right)} e^{-2 \pi i 2\left(g \beta^{\prime}-\alpha_{0}\right)\left(g \beta-\alpha_{0}\right)} \sum_{p^{\prime} \in \Gamma^{\prime}} e^{2 \pi i \tau^{\prime}\left(g \beta^{\prime}+p^{\prime}-\alpha_{0}\right)^{2}} \\
\quad=\frac{1}{\eta\left(\tau^{\prime}\right)^{d}} \sum_{\beta^{\prime} \in\left(\frac{\Gamma}{\Gamma^{\prime}}\right)} e^{-2 \pi i 2\left(\beta^{\prime}-\alpha_{0}\right)\left(\beta-\alpha_{0}\right)} L\left(g \beta^{\prime}, \tau^{\prime}\right)
\end{gathered}
$$

where we have used the fact that $2\left(g \beta^{\prime}-\alpha_{0}\right)\left(g \beta-\alpha_{0}\right)=2\left(\beta^{\prime}-\alpha_{0}\right)\left(\beta-\alpha_{0}\right)$ mod. integers. This can be seen by using the fact that for $g_{a}$

$$
\begin{gathered}
\left(g_{a} \beta-\alpha_{0}\right)^{2}-\left(\beta-\alpha_{0}\right)^{2}=\left(\beta-\alpha_{0}+n_{a} e_{a}\right)^{2}-\left(\beta-\alpha_{0}\right)^{2} \\
=2 n_{a} e_{a}\left(\beta-\alpha_{0}\right)+n_{a}^{2} e_{a}^{2}=n_{a} m_{a}
\end{gathered}
$$


where

$$
\beta e_{a}=\frac{1-n_{a}}{2} e_{a}^{2}-\frac{1-m_{a}}{2}
$$

Using this equation for $\beta, \beta^{\prime}$ and $\beta+\beta^{\prime}$ we see that the above assertion is correct. Inserting eq $(2.9)$ in $(2.7)$

$$
\chi_{[\beta]}(\tau) \rightarrow \sum_{\beta^{\prime} \in\left(\frac{\Gamma}{\Gamma^{\prime}}\right)} e^{-2 \pi i 2\left(\beta^{\prime}-\alpha_{0}\right)\left(\beta-\alpha_{0}\right)} \chi\left[\beta^{\prime}\right]\left(\tau^{\prime}\right)
$$

Now we can construct a modular invariant combination of the characters:

$$
Z=\frac{1}{[\tilde{G}]} \sum_{[\beta] \in \frac{\Gamma}{\Gamma^{\prime}}}\left|\chi_{[\beta]}\right|^{2}
$$

where $[\tilde{G}]$ is the order of $\tilde{G} . Z$ can be rewritten as:

$$
Z=\frac{1}{[\tilde{G}]} \sum_{[\beta] \in \frac{\left(\frac{\Gamma}{\Gamma^{\prime}}\right)}{\tilde{G}}} N(\beta)\left|\chi_{[\beta]}\right|^{2}
$$

where $N(\beta)$ is the number of elements in the orbit and $\chi_{[\beta]}$ can be further rewritten as:

$$
\begin{gathered}
\chi_{[\beta]}=\sum_{g \in \tilde{G}} c(g) L(g \beta) \\
=\frac{[\tilde{G}]}{N(\beta)} \sum_{g \in \frac{\tilde{G}}{G_{\beta}}} c(g) L(g \beta) \\
\equiv \frac{[\tilde{G}]}{N(\beta)} \tilde{\chi}_{[\beta]}
\end{gathered}
$$

(if $c(g)=+1$ for $g \in G_{\beta}$ otherwise $\chi_{[\beta]}=0$ ) where $G_{\beta}$ is the subgroup of $\mathrm{G}$ that leaves $\beta$ invariant. Inserting this in eq. (2.11) we have:

$$
Z=\sum_{[\beta] \in \frac{\left(\frac{\Gamma}{\Gamma^{\prime}}\right)}{\tilde{G}}} \frac{[G]}{N(\beta)}\left|\tilde{\chi}_{[\beta]}\right|^{2}
$$

Let us denote by $n(\beta)$ the dimension of the orbit of $[\beta]$ classes under the action of $G_{0}$. In order to have an unique ground state we must divide $Z$ by $\frac{[G] n^{2}(0)}{N(0)}$. So finally:

$$
Z=\sum_{[\beta] \in \frac{\left(\frac{\Gamma}{\Gamma^{\prime}}\right)}{\tilde{G}}} \frac{N(0)}{N(\beta) n^{2}(0)}\left|\tilde{\chi}_{[\beta]}\right|^{2}
$$


For this partition function to be well defined we must demand that: $\frac{N(0) n^{2}(\beta)}{N(\beta) n^{2}(0)}$ is integer for all $[\beta]$. In particular in the following we shall often use normalized characters: $\hat{\chi}_{[\beta]}$ in which we factor out the action of $G_{0}$. In term of these characters the partition function is:

$$
Z=\sum_{[\beta] \in \frac{\left(\frac{\Gamma}{\Gamma^{\prime}}\right)}{\tilde{G}}} \frac{N(0) n^{2}(\beta)}{N(\beta) n^{2}(0)}\left|\hat{\chi}_{[\beta]}\right|^{2}
$$

We remark that the characters for some of the $\beta$ 's are identically zero. For example for $\beta=\alpha_{0}, g_{a}:\left[\alpha_{0}\right] \rightarrow\left[\alpha_{0}\right]$ and hence $\chi_{\left[\alpha_{0}\right]}=0$

As our model is a Virasoro model,$\chi_{[\beta]}$ for $c>1$ would contain infinite numbers of Virasoro modules. Thus $\chi_{[\beta]}$ should be expressible as:

$$
\chi_{[\beta]}=\frac{q^{h_{\beta}-\frac{c}{24}}}{\eta}\left(1+\sum_{n=1}^{\infty} a_{n} q^{n}\right) .
$$

where all the $a_{n}$ 's must be positive except in the case of the identity sector $(\beta=0) a_{1}$ can be -1 due to the fact that $L_{-1} \mid 0>$ is always a null state. In general the characters we have constructed will not satisfy this stringent condition. Therefore we must select only those models which fulfil this condition. Due to the fact that this check requires an expansion of $\frac{1}{\eta^{d-1}}$ whose coefficients in q expansion are positive and grow rapidly, this condition can be easily checked by looking at the first few terms. We shall show in sect 3 that there are several classes of models satisfying all these conditions and they turn out to correspond to the spin $\frac{4}{3}$ parafermionic and other known series.

In some special cases c can also be less than 1 . In this case the positivity condition must be checked after expanding $\frac{1}{\eta^{d}}$. and the character must be exactly equal to the know one.

The condition we have stated above are sufficient for characters, however as we shall see in the following, in order to be able to construct well defined correlation functions we will need the following condition:

$$
\sum_{a} r_{a} e_{a}=0
$$

for some positive integers $r_{a}$. Furthermore to construct genus 2 characters when one expects a background charge $+2 \alpha_{0}$ we shall need the following condition:

$$
\sum_{a} r_{a} e_{a}=2 \alpha_{0}
$$

in order to balance this charge with screening operators 


\section{B] CORRELATION FUNCTIONS}

In order to give a complete description of a RCFT we must also give all the 4-pt. correlation functions. The fusion rules can be determined by using Verlinde conjecture [4,14] which relates the fusion rule coefficients $N_{i j k}$ with the modular transformation matrices. In order to extract the operator algebra coefficients $c_{i j k}$ one would like to construct 4pt. functions. As our characters were built by subtracting the first level null states using the screening operators one should be able to give an integral representation of the 4-pt. functions using these screening operators. However the characters have an extra input, namely the lattice sum $\Gamma^{\prime}$ and in fact these characters in general cannot be constructed using the screening operators via Feigin-Fuchs procedure. Indeed we shall see that in several examples in the lattice sum $\sum_{g} \Gamma(g \beta)$ there are several states at the lowest level in different g-sectors. These states clearly cannot be thought of as null states of the primary state. The link between the characters we have constructed and the 4-pt. functions on the sphere is obtained by considering 2-pt. functions on the torus. The limit of two points coinciding should reproduce the characters, whereas the degeneration of the torus should reproduce the 4-pt. function on the sphere. These 2-pt. functions must also form a representation of the monodromy and modular group. This could ensure the symmetry and associativity of the resulting $c_{i j k}$ 's upon pinching the torus.

Let us define the set $\hat{G}_{\alpha} \subset \frac{G}{G_{0}}$ for every $\alpha \in \Gamma$ as follows:

$$
\hat{G}_{\alpha}=\left\{g \in \frac{G}{G_{0}} \mid h(g \alpha)=h(\alpha)\right\}
$$

where $h(\alpha)$ is the weight of $\alpha$. We first consider 2-pt functions on the torus $<\phi_{\alpha}\left(z_{1}\right) \phi_{\alpha}\left(z_{2}\right)>$ for fields $\phi_{\alpha}$ such that $\hat{G}_{\alpha}$ is identity. The 2-pt. functions of this type were constructed in ref. [15]. The basic point was that whereas on the sphere one puts a charge $-2 \alpha_{0}$ at $\infty$, on the torus there is no background charge due to the fact that Euler number of the torus is zero. One then uses the vertex operator representation for the $\phi_{\alpha}$ 's and balances the charge by inserting sufficient number of screening operators. Thus the 2-pt. function is:

$$
<\phi_{\alpha}\left(z_{1}\right) \phi_{\alpha}\left(z_{2}\right)>_{\beta}=<V_{\alpha}\left(z_{1}\right) V_{\alpha}\left(z_{2}\right) \prod_{i} \oint_{C_{i}} d w_{i} V_{e_{a_{i}}}\left(w_{i}\right)>_{\beta}
$$

such that $2 \alpha+\sum_{i} e_{a_{i}}=0$ and $\beta$ here denotes that the trace is over the representation over $\beta$. the contours $C_{i}$ are chosen by considering the degeneration limit: $\tau \rightarrow i \infty, z \rightarrow \frac{\log x}{2 \pi i}$ and the two ends of the parallelogram go to $x=0$ and $x=\infty$ (see fig.1). In the s-channel description each of the contours $C_{i}$ is one of the two contours shown in fig. 2 , whereas in the t channel they are one of the two contours shown in fig.3. The 2-pt. function then can be computed using the free field representation. There are two parts: firstly the oscillator contribution which give $\theta$-functions and secondly the lattice contribution (i.e. solitonic states) which get modified in the presence of the vertex operators in the usual way by terms linear in the 
position of the vertex operators

$$
\begin{aligned}
<\phi_{\alpha}\left(z_{1}\right) \phi_{\alpha}\left(z_{2}\right)>_{\beta} & =\left(\frac{\theta\left(z_{1}-z_{2}\right)}{\theta^{\prime}(0)}\right)^{2 \alpha^{2}} \prod_{i=1}^{2} \prod_{j}\left(\frac{\theta\left(z_{i}-w_{j}\right)}{\theta^{\prime}(0)}\right)^{2 \alpha e_{a_{j}}} \\
& \prod_{i>j}\left(\frac{\theta\left(w_{i}-w_{j}\right)}{\theta^{\prime}(0)}\right)^{2 e_{a_{i}} e_{a_{j}}} \frac{1}{\eta^{d}} \sum_{g} \prod_{g\left(C_{i}\right)} d w_{i} c(g) \tilde{\Gamma}(g \beta)
\end{aligned}
$$

where:

$$
\tilde{\Gamma}(g \beta)=\sum_{p \in \Gamma^{\prime}} q^{\left(p+g \beta-\alpha_{0}\right)^{2}} e^{4 \pi i\left(p+g \beta-\alpha_{0}\right)\left(\alpha z_{1}+\alpha z_{2}+\sum_{i} e_{a_{i}} w_{i}\right)}
$$

and $g\left(C_{i}\right)$ will be defined in the following. Let us consider s-channel basis for $C_{i}$. Then if we move $z_{i}$,say, around an a-cycle, this would amount to $x_{1}$ moving around origin once in the annulus picture. This action must be diagonal in the s-channel and it should pick a phase given by $e^{-2 \pi i(h(\beta)-h(I))}$ where $\mathrm{I}$ is the intermediate state. Those $C_{i}$ 's which go from 0 to $x_{1}$, will also be pulled around origin once. This means that one can get the phase by the transformation $z_{1} \rightarrow z_{1}+1, w_{i} \rightarrow w_{i}+1$ for all the $C_{i}$ 's which go around $z_{1}$. Let us denote by $i$ and $\tilde{i}$ the $C_{i}$ 's which go from 0 to $x_{1}$ and from $x_{2}$ to $\infty$ respectively. Then by a simple combinatorics we obtain for the $g=1$ term that the phase is $e^{-2 \pi i(h(\beta)-h(I))}$ where the intermediate state $\mathrm{I}$ is $\beta^{\prime} \equiv\left(\beta+\alpha+\sum e_{a_{i}}\right)$. In general some of these intermediate channels will vanish, provided one or more of the contours have no branch cut at one of the four points $0, z_{1}, z_{2}, \infty$ ( 0 and $\infty$ are the point on the sphere obtained by pinching the torus). However this will depend on the details of the model and we shall illustrate it in some of the examples. For $g \neq 1$ the contour assignement $g\left(C_{i}\right)$ should be such that $\beta^{\prime \prime} \equiv\left(g \beta+\alpha+\sum e_{a_{g(i)}}\right)=g \beta^{\prime}$ $\bmod \frac{1}{2} \Gamma^{*}$. This would also ensure that under the transformation $z_{1} \rightarrow \tau-z_{1}$, the character and intermediate state interchanges, namely $\chi_{\beta} \rightarrow \chi_{\beta^{\prime}}$. First let us consider the subgroup of $\tilde{G}$ generated by the screening operators. For $g=g_{a}$ the above condition means:

$$
\sum e_{a_{g(i)}}=\sum e_{a_{i}}+n e_{a} \quad \bmod \frac{1}{2} \Gamma^{*}
$$

where $2\left(\alpha+\sum e_{a_{i}}\right) e_{a}=n e_{a}^{2} \bmod$ integers. Thus in $g_{a}$ sector the number of $e_{a}$ contours going from 0 to $\mathrm{z}$, is increased by $\mathrm{n}$. If the total number of $e_{a}$ type of screening operators in the correlation function is $N_{a}$ and if in $g=1$ sector we start with $n_{a}$ of these contours running from 0 to $z_{1}$, then this condition can only be satisfied provided $n_{a}+n \leq N_{a}$. However if there exist positive integers $r_{a}$ such that $\sum_{a} r_{a} e_{a}=0$ then clearly we can replace $N_{a}$ by $N_{a}+r_{a}$ and the above condition could then be satisfied (note that $\mathrm{n}$ can be taken to be less than $k_{a}$ ). We shall therefore further impose the requirement that exist positive integers $r_{a}>0$ such that:

$$
\sum r_{a} e_{a}=0
$$

This condition would then allow one to assign contours for each element of the subgroup $\tilde{G} / G_{0}$ where $G_{0}$ is the dimension preserving symmetry group (not generated by the action of screening operators). 
The action of $G_{0}$ is more subtle. In general there would be no solution to eq.(2.15). We therefore have to modify the torus propagator as follows:

$$
<\phi_{\alpha}\left(z_{1}\right) \phi_{\alpha}\left(z_{2}\right)>_{\beta}=\sum_{g \in G_{0}}<\phi_{g \alpha}\left(z_{1}\right) \phi_{g \alpha}\left(z_{2}\right)>_{g \beta}^{\prime}
$$

where $<. .>_{g \beta}^{\prime}$ denotes the restricted lattice sum:

$$
\sum_{g^{\prime} \in \tilde{G} / G_{0}} \Gamma\left(g^{\prime} g \beta\right) c\left(g^{\prime}\right)
$$

note that for different $g \in G_{0}$ one may need different numbers of screening operators in eq(2.16) to saturate the charges. To obtain the 4-pt function on the sphere one degenerates the torus by the limit $\tau \rightarrow i \infty$ and extract all the terms in eq.(2.16) coming with the power: $q^{h(\beta)-\frac{c}{24}}$.

In the above we had assumed that $\hat{G}_{\alpha}$ was identity. However when $\hat{G}_{\alpha}$ is also non trivial, in order to ensure the symmetry of the structure constants we must also sum over $g^{\prime \prime} \alpha, g^{\prime \prime} \in \hat{G}_{\alpha}$ in the above expression. However in general these different terms may require different numbers of screening operators and therefore their relative normalizations can be fixed only by studying monodromy properties. On the other hand if one could extend the definition of characters to genus 2 then these relative normalizations would be fixed. However we shall not go into this here.

In the multicontour problem, in general one can face the problem of ordering of contours. This happens when there are two screening charges $e_{a}$ and $e_{b}$ such that $2 e_{a} e_{b}$ is a negative integer (say -n) as:

$$
<. . V_{e_{a}}(x) V_{e_{b}}(y)>=\int d x d y(x-y)^{-n} f(x, y) .
$$

A change in the ordering of the contours of $e_{a}$ and $e_{b}$ would pick the residue

$$
\left.\int d y\left(\partial_{x}^{n-1} f(x, y)\right)\right|_{x=y}
$$

If $n=1$ this residue is clearly non zero therefore we must discard those models. When $\mathrm{n}=2$, however, for special forms of $f(x, y),\left.\left(\partial_{x} f(x, y)\right)\right|_{x=y}$ can be a total derivative. Indeed this is what happens for the one boson Coulomb gas models $(c<1)$ as shown by Dotsenko and Fateev [9], as well as for the 2-bosons models studied by Fateev and Zamolodchikov [11]. One can construct more general 2-bosons models that also share this property by introducing a $Z_{2}$ symmetry as a part of $G_{0}$. Let $e_{1}, e_{2}, e_{3}, e_{4}$ be the four screening charges such that $e_{2}=\frac{2 \alpha_{0} e_{1}}{\alpha_{0}^{2}} \alpha_{0}-e_{1}, e_{3}=2 \alpha_{0}-e_{1}$, and $e_{4}=2 \alpha_{0}-e_{2}$. These screening charges have clearly a $Z_{2}$ symmetry corresponding to the reflection $\mathrm{R}$ about the $\alpha_{0}$ line. Consequently the lattice $\Gamma$ and $\Gamma^{*}$ will also have this $Z_{2}$ symmetry. Now consider a correlation function for which one needs one each of $V_{e_{1}}$ and $V_{e_{3}}$ screening charges to balance the charge:

$$
<\prod_{i} V_{\beta_{i}}\left(z_{i}\right) V_{e_{1}}(x) V_{e_{3}}(y)>
$$


Summing over the action of the above $Z_{2}$ symmetry group (as point of $G_{0}$ ) and using the fact that $R e_{1}=e_{2}, R e_{3}=e_{4}$ we obtain:

$$
\iint d x d y \prod_{i}\left(x-z_{i}\right)^{a_{i}} \prod_{i}\left(y-z_{i}\right)^{b_{i}}\left[\prod_{i}\left(\frac{x-z_{i}}{y-z_{i}}\right)^{c_{i}}+\prod_{i}\left(\frac{y-z_{i}}{x-z_{i}}\right)^{c_{i}}\right](x-y)^{-2}
$$

where:

$$
\begin{gathered}
a_{i}=\left(e_{1}+e_{2}\right) \beta_{i} \\
b_{i}=\left(e_{3}+e_{4}\right) \beta_{i}=\left(\frac{2 \alpha_{0}^{2}}{\alpha_{0} e_{1}}-1\right) a_{i} \\
c_{i}=\left(e_{1}-e_{2}\right) \beta_{i}=-\left(e_{3}-e_{4}\right) \beta_{i} .
\end{gathered}
$$

Now suppose both the contours are from, say, $z_{1}$ to $z_{2}$. Then changing the ordering we would obtain the difference:

$$
\begin{gathered}
\left.\int d y \prod_{i}\left(y-z_{i}\right)^{b_{i}}\left\{\partial_{x} \prod_{i}\left(x-z_{i}\right)^{a_{i}}\left[\prod_{i}\left(\frac{x-z_{i}}{y-z_{i}}\right)^{c_{i}}+\prod_{i}\left(\frac{y-z_{i}}{x-z_{i}}\right)^{c_{i}}\right]\right\}\right|_{x=y} \\
=\frac{\alpha_{0} e_{1}}{2 \alpha_{0}^{2}} \int_{z_{1}}^{z_{2}} d y \frac{d}{d y} \prod_{i}\left(y-z_{i}\right)^{a_{i}+b_{i}}=0
\end{gathered}
$$

where we have assumed that the integrand does not get contribution from the end points. Thus we see that for a large class of 2-bosons models one can ensure that the integrals do not depend on ordering of contours. In the next section we shall be mainly studying examples of this type. In more complicated examples where one does not have this $Z_{2}$ symmetry (one such example will be analyzed in the next section) or for the theories involving more than 2 bosons we do not have a general prescription for this and they have to be studied case by case.

To construct explicitely the monodromy invariant combinations of left and right conformal blocks for correlators is very difficult in practice although one can do this for examples involving few screening operators by following the procedure of Dotsenko and Fateev [9]. However by going through 2-dimensional integrals rather than contour integrals one would obtain monodromy invariant correlators [16]. Splitting the 2-dimensional integrals into contour integrals one can then obtain the monodromy invariant combination of conformal blocks. We shall here not construct such combinations, however the possibility of using 2-dimensional integrals prove in principle the existence of monodromy invariant correlators. 


\section{Section 3: Examples: characters}

In this section we give some examples with 2 bosons of the previous construction. Obvious examples are the SU(3) models of Fateev and Zamolodchikov [11]. In fact one could check that the characters obtained following the prescriptions of sect.2 coincide with the ones constructed using Feigin-Fuchs procedure [17], as well as using GKO construction [13] Our strategy to construct new examples is the following: starting from the screening charges we first identify $\Gamma, \Gamma^{\prime}$ and describe the $\left(\frac{\Gamma}{\Gamma^{\prime}}\right)$ conjugacy classes; then we find the $g_{a}$ 's and $g_{i}$ 's operations, discuss the group structure and describe the classes $\frac{\left(\Gamma / \Gamma^{\prime}\right)}{\tilde{G}}$ (namely the primary fields of the theory). Finally we write characters and partition function of the model. The main problem we have to face is that even in two dimension we have a huge number of possible choices of the screening charges so the first step is to reduce this number: we devote the first part of this section to this point. We want to stress that there will be some arbitrariness in each one of the four requirements that we shall impose in the following and that a complete classification of all the 2-bosons models is far to be obtained .

\section{A] Screening charges}

We proceed as follows: first we reduce the set of all possible screening charges by using very general requirements: we obtain in this way the spin 4/3 Fateev-Zamolodchikov models [18] and the series described by the GKO coset:

$$
\frac{S U(2)^{k} \times S U(2)^{1} \times S U(2)^{1}}{S U(2)^{k+2}}
$$

then, ignoring the last one of these constraints we obtain two of the models of the

$\frac{S U(3)^{k} \times S U(3)^{1}}{S U(3)^{k+1}}$ series [11] namely the $\mathrm{c}=4 / 5$ and the $\mathrm{c}=10 / 7$ models. The interesting feature of these last examples is the presence of additional symmetries in the lattice $\Gamma$ of allowed states. Finally we present an example with asymmetric screening charges which turns out to be equivalent to the $\mathrm{c}=-22 / 5$ non unitary model (Lee-Yang edge-singularity model) of the Virasoro series [19].

We choose $\vec{\alpha}_{0}$ as:

$$
\left(\begin{array}{c}
\alpha_{0} \\
0
\end{array}\right)
$$

and for future convenience and to make contact with the usual convention we shift the lattice $\Gamma$ adding $\vec{\alpha}_{0}$, the only change in the analysis of sect. 2 is in the weight: given any state $\beta$ is:

$$
h=\beta^{2}-\alpha_{0}^{2}
$$


i] We choose for the screening charges the following symmetrical expression:

$$
\left(\begin{array}{c}
\alpha_{0} \pm a \\
\pm b
\end{array}\right)
$$

(Note that with this choice we rule out, say, the Fateev-Zamolodchikov approach to the $\frac{S U(3)^{k} \times S U(3)^{1}}{S U(3)^{k+1}}$ models, nevertheless due to non trivial identities between the lattices of allowed states in the two approaches we shall be able at the end of this section to obtain 2 of these models)

ii] In order to ensure that the screening charges belong to the set $\Gamma$ of the allowed states we require:

$$
a=\frac{k}{2} \alpha_{0} \quad(k \in \mathbf{Z})
$$

iii] Then in order to avoid states with negative weight we require, at the same time:

$$
\alpha_{0}^{2}=\frac{1}{(k+2) t}=\frac{1}{(k-2) t^{\prime}} \quad\left(t, t^{\prime} \in \mathbf{Z}\right)
$$

These conditions can be satisfied with the following parametrization:

$$
4 t=l^{\prime}(k-2), 4 t^{\prime}=l^{\prime}(k+2)
$$

then:

$$
b^{2}=\frac{l^{\prime}-1}{l^{\prime}}
$$

Let us now examine $\mathrm{k}$ : we have the following classification:

a] k odd: $k=2 n+1$ then $4 t=l^{\prime}(2 n-1)$, define $l^{\prime}=4 l$, then $b^{2}=\frac{4 l-1}{4 l}$ and:

$$
\alpha_{0}^{2}=\frac{1}{\left(k^{2}-4\right) l} \quad(k \quad \text { odd }, \quad l \in \mathbf{N})
$$

hence:

$$
c=2-\frac{24}{\left(k^{2}-4\right) l}
$$

b] k even: $\mathrm{k}=4 \mathrm{n}+2$, then $t=l^{\prime} n$, hence $b^{2}=\frac{l-1}{l}$ and:

$$
c=2-\frac{6}{n(n+1) l^{\prime}}
$$


c] k even: $\mathrm{k}=4 \mathrm{n}$, then $2 t=l^{\prime}(2 n-1) ; l^{\prime}=2 l$, hence $b^{2}=\frac{2 l-1}{2 l}$ and:

$$
c=2-\frac{12}{(2 n-1)(2 n+1) l}
$$

iv] Finally, it turns out that a sufficient condition for the last requirement in the previous section on the positivity of the characters is that $b^{2}$ in the screening charge must be a rational number of the type: $\frac{r}{4}, r \in \mathbf{Z}$ this selects $\mathrm{l}=1$ if $\mathrm{k}$ is odd, $\mathrm{l}=1,2$ if $\mathrm{k}=4 \mathrm{n}, \mathrm{l}=2,4$ if $\mathrm{k}=4 \mathrm{n}+2$

The correspondence between these series and the known models is the following:

1] the GKO coset for the spin $4 / 3$ models is $\frac{S U(2)^{p} \times S U(2)^{4}}{S U(2)^{p+4}}$ : the odd values of $\mathrm{p}$ are given in our construction by the $\mathrm{k}$ odd, $\mathrm{l}=1$ models, $\mathrm{p}=4 \mathrm{n}$ are given by $\mathrm{k}=4 \mathrm{n}, \mathrm{l}=2$ and $\mathrm{p}=4 \mathrm{n}+2$ are given by $\mathrm{k}=4 \mathrm{n}+2, \mathrm{l}=4$. The common feature of all these series is that $b^{2}=\frac{3}{4}$.

2] the $\frac{S U(2)^{p} \times S U(2)^{1} \times S U(2)^{1}}{S U(2)^{p+2}}$ models correspond to $\mathrm{k}=4 \mathrm{n}+2, \mathrm{l}=2$ if $\mathrm{p}$ is even and $\mathrm{k}=4 \mathrm{n}$, $\mathrm{l}=1$ if $\mathrm{p}$ is odd. the common feature of these two series is that $b^{2}=\frac{1}{2}$.

B] the 4/3 parafermionic series

We examine in detail the series with odd values of $\mathrm{k}$. Looking at the screening operators we see that there is a natural division in two sectors which we label with + and - The screening charges are (we write them for general $l$ for future convenience, notice that in this case $l=1)$ :

$$
\begin{aligned}
e_{1}^{+}=\left(\begin{array}{c}
\frac{1}{2 \sqrt{l}} \sqrt{\frac{k+2}{k-2}} \\
b
\end{array}\right), & e_{2}^{+}=\left(\begin{array}{c}
\frac{1}{2 \sqrt{l}} \sqrt{\frac{k+2}{k-2}} \\
-b
\end{array}\right) \\
e_{1}^{-}=\left(\begin{array}{c}
-\frac{1}{2 \sqrt{l}} \sqrt{\frac{k-2}{k+2}} \\
b
\end{array}\right), & e_{2}^{-}=\left(\begin{array}{c}
-\frac{1}{2 \sqrt{l}} \sqrt{\frac{k-2}{k+2}} \\
-b
\end{array}\right)
\end{aligned}
$$

with $b^{2}=\frac{3}{4}$

Let us now impose eq(2.3) say in the plus sector:

$$
\begin{aligned}
& \beta e_{1}^{+}=\frac{1+n_{1}^{+}}{2}\left(e_{1}^{+}\right)^{2}-\frac{1+m_{1}^{+}}{2} \\
& \beta e_{2}^{+}=\frac{1+n_{2}^{+}}{2}\left(e_{2}^{+}\right)^{2}-\frac{1+m_{2}^{+}}{2}
\end{aligned}
$$


the solution of these equations is:

$$
\beta^{+}=\left(\begin{array}{c}
\left\{\left(m_{1}^{+}+m_{2}^{+}-n_{1}^{+}-n_{2}^{+}\right)(k-2)+\left(m_{1}^{+}+m_{2}^{+}\right)\right\} \frac{\alpha_{0}}{2} \\
\left\{m_{1}^{+}-m_{2}^{+}+n_{1}^{+}-n_{2}^{+}+\frac{m_{1}^{+}-m_{2}^{+}}{(k-2)}\right\} \frac{1}{4 b}
\end{array}\right)
$$

The same condition in the minus sector gives:

$$
\beta^{-}=\left(\begin{array}{c}
\left\{\left(n_{1}^{-}+n_{2}^{-}-m_{1}^{-}-m_{2}^{-}\right)(k+2)+\left(m_{1}^{-}+m_{2}^{-}\right)\right\} \frac{\alpha_{0}}{2} \\
\left\{m_{1}^{-}-m_{2}^{-}+n_{1}^{-}-n_{2}^{-}+\frac{m_{2}^{-}-m_{1}^{-}}{(k+2)}\right\} \frac{1}{4 b}
\end{array}\right)
$$

$n_{i}^{ \pm}, m_{i}^{ \pm}$must be positive integers, but notice that we could at this point repeat the same analysis looking at the null states of $V_{2 \alpha_{0}-\beta}$ :

$$
\prod_{i=1}^{n} \oint d z_{i} V_{e_{1,2}^{ \pm}}\left(z_{i}\right) V_{2 \alpha_{0}-\beta-n_{1,2}^{ \pm} e_{1,2}^{ \pm}}(0)
$$

and we reobtain all the previous results with all the signs of $n_{1,2}^{ \pm}, m_{1,2}^{ \pm}$reverted.

Since we are looking for a completely degenerate representation we must sort out the intersection $\left\{\beta^{+}\right\} \bigcap\left\{\beta^{-}\right\}$. In general this will give some relation between $\left(n_{1}^{+}, m_{1}^{+}\right)$and $\left(n_{1}^{-}, m_{1}^{-}\right)$and from this possibly some further constraint on the allowed states. In this case it is easy to see that the condition is:

$$
\begin{gathered}
m_{1}^{+}-m_{2}^{+}=r(k-2)(r \in \mathbf{Z}) \\
m_{1}^{-}-m_{2}^{-}=r^{\prime}(k+2)\left(r^{\prime} \in \mathbf{Z}\right)
\end{gathered}
$$

Taking into account this last requirement we see that a good basis for the set $\Gamma$ of completely degenerate states is

$$
\left\{\left(\begin{array}{c}
0 \\
\frac{1}{2 b}
\end{array}\right),\left(\begin{array}{c}
\frac{\alpha_{0}}{2} \\
\frac{1}{4 b}
\end{array}\right)\right\}
$$

The lattice $\Gamma^{\prime}$ is generated by:

$$
\left\{\left(\begin{array}{c}
\frac{1}{\alpha_{0}} \\
0
\end{array}\right),\left(\begin{array}{c}
\frac{1}{2 \alpha_{0}} \\
b
\end{array}\right)\right\}
$$

Using the fact that 3 and 4 are coprimes we can parametrize the $\left(\frac{\Gamma}{\Gamma^{\prime}}\right)$ conjugacy classes as follows:

$$
\beta(x, y, s)=\left(\begin{array}{c}
0 \\
\frac{s}{2 b}
\end{array}\right)+\left(\begin{array}{c}
x \frac{\alpha_{0}}{2} \\
y b
\end{array}\right)
$$

with $x=0, \pm 1, \pm 2, \ldots, k^{2}-4 ; y=0$ if $x$ is even, $y=1$ if $x$ is odd ; $s=0, \pm 1$.

Next step is to find the $g_{1,2}^{ \pm}$which encode the informations on the null states. One can check by direct calculation that, in the previous parametrization $\mathrm{s}$ is not affected by the action of $\mathrm{g}$ and that the action on $x$ and $y$ is given by the following matrices: 


$$
\begin{aligned}
g_{1}^{+}=\left(\begin{array}{cc}
\frac{k}{2} & -\frac{k^{2}-4}{2} \\
\frac{1}{2} & -\frac{k}{2}
\end{array}\right) & g_{2}^{+}=\left(\begin{array}{cc}
\frac{k}{2} & \frac{k^{2}-4}{2} \\
-\frac{1}{2} & -\frac{k}{2}
\end{array}\right) \\
g_{1}^{-}=\left(\begin{array}{cc}
-\frac{k}{2} & -\frac{k^{2}-4}{2} \\
\frac{1}{2} & \frac{k}{2}
\end{array}\right) & g_{2}^{-}=\left(\begin{array}{cc}
-\frac{k}{2} & \frac{k^{2}-4}{2} \\
-\frac{1}{2} & \frac{k}{2}
\end{array}\right)
\end{aligned}
$$

The $g_{i}$ 's (namely the operations which describe the symmetries of the lattice $\Gamma$ ) involve also $s$ but are very simple to handle since in this case $\Gamma$ has only the trivial symmetries: let us call them $g_{u p}$ and $g_{d o w n}$ :

$$
\begin{gathered}
g_{\text {up }}: x \rightarrow-x \\
g_{\text {down }}:(y, s) \rightarrow(-y,-s)
\end{gathered}
$$

In this case the group structure is rather simple (for instance one can check by using $\Gamma^{\prime}$ and $g_{u p}$ that all the $g_{1,2}^{ \pm}$are equivalent). In order to identify the $\frac{\left(\Gamma / \Gamma^{\prime}\right)}{\tilde{G}}$ classes is useful to have a simpler expression of the action of $g_{1,2}^{ \pm}$, which can be obtained writing $x$ as:

$$
x=\frac{n(k-2)-m(k+2)}{2}
$$

with $n=0, \pm 1, \ldots, \pm(k+1)$ and $m=0, \pm 1, \ldots, \pm(k-3)$ (n and $m$ are both even or both odd). then the action of $g_{1,2}^{ \pm}$on $x$ is:

$$
n(k-2)-m(k+2) \rightarrow n(k-2)+m(k+2) \quad\left(\bmod \Gamma^{\prime}\right)
$$

while $y$ is determined by the parity of $x$. We can label the classes (taking into account $g_{u p}$ and $\left.g_{\text {down }}\right)$ with $\left(k^{2}-4\right) / 2$ positive values of $\mathrm{s}, \mathrm{n}, \mathrm{m}$; but notice that as expected some of this classes (namely the ones in which $\mathrm{n}$ and/or $\mathrm{m}$ is zero) are even under the action of $\tilde{G}$ hence the corresponding characters vanish identically and we end up with $\frac{(k-3)(k+1)}{2}$ independent primary fields. Finally notice that using this notation we have introduced a "conformal grid" symmetry: $(k+2-n, k-2-m)$ and $(n, m)$ describe the same state.

We can at this point look in more detail at the correspondence with the

$4 / 3$ parafermionic model: we can write the weights (namely $\beta(x, y, s)^{2}-\alpha_{0}^{2}$ ) as

$$
h(n, m, s)=\alpha_{0}^{2}\left\{\left(\frac{n(k-2)-m(k+2)}{16}\right)^{2}-1\right\}+h(y, s)
$$

where $\mathrm{h}(\mathrm{y}, \mathrm{s})$ is given as follows: if $x$ is even (hence $n-m=0(\bmod 4))$ and if $y+\frac{2 s}{3}=0$ then $h(y, s)=0$, (singlet state) if $y+\frac{2 s}{3}=\frac{2}{3}$ then $h(y, s)=\frac{1}{3}$ (doublet) and these are exactly the $\mathrm{S}$ fields of the spin $\frac{4}{3}$ model

If $x$ is odd (hence $n-m=2(\bmod 4)$ the lowest weight state is a doublet: $y+\frac{s}{3}=\frac{1}{3}$ for which is $h(y, s)=\frac{1}{12}$ while if $y+\frac{s}{3}=1$ we have : $h(y, s)=\frac{3}{4}$ and these are exactly the D fields of the spin $4 / 3$ models.

In some exceptional case some of these states disappear. This is the case for instance of the identity where we have no $h=\frac{1}{3}$ states. This feature has been noticed by Fateev and Zamolodchikov [18] and will play a major role in the correlation function calculation. 
As it is well known the S and D fields form a closed subalgebra and have modular invariant partition functions.

Following the recepy given in sect.2 we can now write the characters:

$$
\begin{gathered}
\chi_{n, m, s}=\frac{1}{\eta^{2}(q)} \sum_{l_{1}, l_{2} \in \mathbf{Z}} q\left\{\left(\begin{array}{c}
\frac{n(k-2)-m(k+2)}{4} \alpha_{0}+\left(l_{1}+l_{2}\right) \frac{\sqrt{k^{2}-4}}{2} \\
\left(y+\frac{2 s}{3}\right) b+\left(l_{1}-l_{2}\right) b
\end{array}\right)\right\}^{2}-\alpha_{0}^{2} \\
\left.-q\left(\frac{n(k-2)+m(k+2)}{4} \alpha_{0}+\left(l_{1}+l_{2}\right) \frac{\sqrt{k^{2}-4}}{2}\right)\right\}^{2}-\alpha_{0}^{2} \\
\left(y+\frac{2 s}{3}\right) b+\left(l_{1}-l_{2}\right) b
\end{gathered}
$$

with $n=1 . . k+1 ; m=1 \ldots(k-3) ; s=0,1 ; n-m$ even

One can check that these characters have the same modular transformations as the known characters [23]. We have not been able to derive an analytic proof of the equivalence between them due to the presence of $2 \eta$ functions at the denominator (while in the usual expression we have only one $\eta$ ). Nevertheless we are completely certain of this equivalence: we made a computer check up to the order $q^{100}$ for all the characters of the $\mathrm{k}=5$ model (which corresponds to the $\mathrm{c}=6 / 7$ model of the Virasoro series) and we found a perfect agreement of the two expansions.

Finally the partition function is given by:

$$
Z=\frac{1}{2} \sum_{s=0}^{1} \sum_{n=1}^{k+2} \sum_{m=1}^{k-2} m(s)\left|\chi_{n, m, s}\right|^{2} \quad(n-m=\text { even })
$$

where $m(0)=1$ and $m(1)=2$ the multiplicity $\mathrm{m}(\mathrm{s})$ being the combination $\frac{N(0) n^{2}(\beta)}{N(\beta) n^{2}(0)}$ defined in the previous section.

We give in the following as an example the details of the simplest model of the series: $k=5, c=\frac{6}{7}$ and the comparision between the usual BPZ formulation and our formulation: Let us introduce a shorthand notation (which we shall use also in sect. 4 in the correlation function calculation):

$$
\left(\begin{array}{l}
x \\
y
\end{array}\right)=\left(\begin{array}{l}
\frac{x}{2 \sqrt{21}} \\
y \sqrt{\frac{3}{4}}
\end{array}\right)
$$

hence the weight of $\left(\begin{array}{l}x \\ y\end{array}\right)$ is:

$$
h(x, y)=\frac{x^{2}-4}{84}+\frac{3}{4} y^{2} .
$$

We have 6 fields in our model: in table 1 we give these fields in three different languages: our one, usual BPZ [1] and in the spin $\frac{4}{3}$ approach [18]. 
Table 1:

In the first column are our characters, in the second column the weights, in the third the corresponding combinations of Virasoro fields in the notation $(\mathrm{r}, \mathrm{s})$ (notice that this model correspond to $\mathrm{m}=6$ ) and in the last column we report the same fields in the language of [18]. The hat indicates the " $\frac{1}{3}$-secondaries" of the primary field (see [18])

$$
\begin{aligned}
\left(\begin{array}{l}
2 \\
0
\end{array}\right)-\left(\begin{array}{l}
5 \\
1
\end{array}\right) & h=0 & (1,1)+(1,6) & S_{(1,1)} \\
\left(\begin{array}{c}
4 \\
0
\end{array}\right)-\left(\begin{array}{c}
10 \\
0
\end{array}\right) & h=\frac{1}{7} & (1,2)+(1,5) & D_{(5,1)} \\
\left(\begin{array}{c}
1 \\
1
\end{array}\right)-\left(\begin{array}{c}
8 \\
0
\end{array}\right) & h=\frac{5}{7} & (1,3)+(1,4) & \hat{D}_{(3,1)} \\
\left(\begin{array}{c}
2 \\
\frac{2}{3}
\end{array}\right)-\left(\begin{array}{c}
5 \\
\frac{1}{3}
\end{array}\right) & h=\frac{4}{3} & (3,1) & \hat{S}_{(1,1)} \\
\left(\begin{array}{c}
4 \\
\frac{2}{3}
\end{array}\right)-\left(\begin{array}{c}
10 \\
\frac{2}{3}
\end{array}\right) & h=\frac{10}{21} & (3,2) & \hat{D}_{(5,1)} \\
\left(\begin{array}{c}
1 \\
\frac{1}{3}
\end{array}\right)-\left(\begin{array}{c}
8 \\
\frac{2}{3}
\end{array}\right) & h=\frac{1}{21} & (3,3) & D_{(3,1)}
\end{aligned}
$$

Notice some non trivial features of these fields in our language:

i] the $h=\frac{5}{7}$ field has 3 components with the same highest weight $h=\frac{5}{7}$ :

$$
\left(\begin{array}{l}
1 \\
1
\end{array}\right), \quad\left(\begin{array}{c}
1 \\
-1
\end{array}\right), \quad\left(\begin{array}{l}
8 \\
0
\end{array}\right)
$$

the first two with a positive sign and the third with a negative sign. As a result of this cancellation this field is a singlet. In correlation function calculations all the fields must be taken into account.

ii] in the case of the field of weight $\frac{4}{3}$ we have that the ground state is at level $\frac{1}{3}$ but the 4 fields:

$$
\left(\begin{array}{c}
2 \\
\frac{2}{3}
\end{array}\right)+\left(\begin{array}{c}
2 \\
-\frac{2}{3}
\end{array}\right)-\left(\begin{array}{c}
5 \\
\frac{1}{3}
\end{array}\right)-\left(\begin{array}{c}
5 \\
-\frac{1}{3}
\end{array}\right)
$$

cancels exactly. Hence the first non zero contribution in the characters comes from:

$$
\left(\begin{array}{l}
2 \\
\frac{4}{3}
\end{array}\right)+\left(\begin{array}{c}
2 \\
-\frac{4}{3}
\end{array}\right)
$$

and is at level $h=\frac{4}{3}$.

iii] The fields of weight $\frac{10}{21}, \frac{1}{21}, \frac{4}{3}$ are doublets. 
Notice that in all these considerations we have ignored the $Z_{2}$ symmetry $g_{u p}$ which acts trivially on all the states (also the identity). This symmetry corresponds to the exchange $\beta \rightarrow 2 \alpha_{0}-\beta$ and has indeed a natural interpretation in the context of the torus correlation functions.

C] the $\frac{S U(2)^{p} \times S U(2)^{1} \times S U(2)^{1}}{S U(2)^{p+2}}$ series

We describe in the following the even $\mathrm{p}$ case. In this case $b^{2}=\frac{1}{2}$ and

$$
\alpha_{0}^{2}=\frac{1}{8 n(n+1)}
$$

where the relation between $\mathrm{n}, \mathrm{p}$ is: $p=2 n-2$ The screening charges are:

$$
\begin{aligned}
e_{1}^{+}=\left(\begin{array}{c}
\frac{1}{\sqrt{2}} \sqrt{\frac{n+1}{n}} \\
b
\end{array}\right), & e_{2}^{+}=\left(\begin{array}{c}
\frac{1}{\sqrt{2}} \sqrt{\frac{n+1}{n}} \\
-b
\end{array}\right) \\
e_{1}^{-}=\left(\begin{array}{c}
-\frac{1}{\sqrt{2}} \sqrt{\frac{n}{n+1}} \\
b
\end{array}\right), & e_{2}^{-}=\left(\begin{array}{c}
-\frac{1}{\sqrt{2}} \sqrt{\frac{n}{n+1}} \\
-b
\end{array}\right)
\end{aligned}
$$

the basis for the lattice $\Gamma$ of allowed states is:

$$
\left\{\left(\begin{array}{c}
0 \\
\frac{1}{4 b}
\end{array}\right),\left(\begin{array}{c}
\alpha_{0} \\
0
\end{array}\right)\right\}
$$

and the basis for $\Gamma^{\prime}$ is:

$$
\left\{\left(\begin{array}{c}
0 \\
2 b
\end{array}\right),\left(\begin{array}{c}
4 n(n+1) \alpha_{0} \\
0
\end{array}\right)\right\}
$$

We can parametrize the $\frac{\Gamma}{\Gamma^{\prime}}$ classes as follows:

$$
\beta(x, y)=\left(\begin{array}{c}
x \alpha_{0} \\
\frac{y}{2} b
\end{array}\right)
$$

with $x=1 \ldots 4 n(n+1) ; y=0, \pm 1,2$ The matrices which encode the null state information are:

$$
\begin{array}{cc}
g_{1}^{+}=\left(\begin{array}{cc}
2 n+1 & 2 n(n+1) \\
-2 & -(2 n+1)
\end{array}\right) & g_{2}^{+}=\left(\begin{array}{cc}
-(2 n+1) & -2 n(n+1) \\
2 & 2 n+1
\end{array}\right) \\
g_{1}^{-}=\left(\begin{array}{cc}
2 n+1 & -2 n(n+1) \\
2 & -(2 n+1)
\end{array}\right) & g_{2}^{-}=\left(\begin{array}{cc}
-(2 n+1) & 2 n(n+1) \\
-2 & 2 n+1
\end{array}\right)
\end{array}
$$


Also in this case the lattice $\Gamma$ has only trivial symmetries hence we have:

$$
\begin{gathered}
g_{\text {up }}: x \rightarrow-x \\
g_{\text {down }}:(z) \rightarrow(-z)
\end{gathered}
$$

and, like in the previous case one can prove that all the $g_{1,2}^{ \pm}$'s are equivalent by using $\Gamma^{\prime}$, $g_{u p}$ and $g_{\text {down }}$. We can split the $\beta$ 's into four classes which have different behaviour under $g_{1,2}^{ \pm}$

$$
\begin{array}{lr}
\text { A] } & \left(\begin{array}{c}
2 k \\
0
\end{array}\right) \rightarrow\left(\begin{array}{c}
2 k(2 n+1) \\
0
\end{array}\right) \\
\text { B] } & \left(\begin{array}{c}
2 k+1 \\
0
\end{array}\right) \rightarrow\left(\begin{array}{c}
(2 k+1)(2 n+1) \\
0
\end{array}\right) \\
\text { C] } & \left(\begin{array}{c}
2 k \\
2
\end{array}\right) \rightarrow\left(\begin{array}{c}
2 k(2 n+1) \\
2
\end{array}\right) \\
\text { D] } & \left(\begin{array}{c}
k \\
1
\end{array}\right) \rightarrow\left(\begin{array}{c}
k(2 n+1)+2 n(n+1) \\
1
\end{array}\right)
\end{array}
$$

Some of these combinations are even under the action of $\mathrm{G}$ and vanish. We end up with $\frac{n(n-1)}{2}$ states of type A, $\frac{n(n-1)}{2}$ of type C, $n(n+1)$ of type $\mathrm{B}$ and $n^{2}$ of type D. So all together we have $3 n^{2}$ states.

Characters are given by:

$$
\chi(x, z)=\frac{1}{\eta^{2}} \sum_{l_{1}, l_{2}}\left\{q^{\alpha\left(l_{1}, l_{2}, x, z\right)}-q^{\alpha\left(l_{1}, l_{2}, \tilde{x}, \tilde{z}\right)}\right\}
$$

where:

$$
\alpha\left(l_{1}, l_{2}, x, z\right)=\frac{\left[l_{1} 4 n(n+1)+x\right]^{2}-1}{8 n(n+1)}+\frac{\left[4 l_{2}+z\right]^{2}}{8}
$$

and $\left(\begin{array}{l}x \\ z\end{array}\right)$ and $\left(\begin{array}{l}\tilde{x} \\ \tilde{z}\end{array}\right)$ are related by eqs. $(1$, a...d).

In Appendix A we shall prove that these characters are equivalent to those obtained by applying GKO construction to the coset

$$
\frac{S U^{p}(2) \times S U^{1}(2) \times S U^{1}(2)}{S U^{p+2}(2)}
$$

in appendix B we shall also prove that these models can be thought as product of an Ising model and a model from the Supersymmetric $(\mathrm{N}=1)$ series by an explicit decomposition of the characters.

The partition function is:

$$
\sum_{(x, z) \in\{A, B, C, D\}} m(z)\left|\chi_{x, z}\right|^{2}
$$


with $m(0)=m(2)=1$ and $m(1)=2$ : the D fields are doublets. Notice that the first model of this series is the Ising model $c=\frac{1}{2}$. We shall describe in detail this new version of the Ising model in sect. 4

D] The $Z_{3}$ symmetric models

$c=\frac{4}{5}$

Let us take the screening charges (3.4) in the case in which $l=4$ and $k=3$. It is easy to see that the corresponding value of $\mathrm{c}$ is $\frac{4}{5}$. The first part of the analysis follows what we did for the spin $\frac{4}{3}$ series with minor changes: now the lattice $\Gamma$ is given by:

$$
\left\{\left(\begin{array}{c}
0 \\
\frac{1}{2 b l}
\end{array}\right),\left(\begin{array}{c}
\frac{\alpha_{0}}{2} \\
\frac{1}{4 b l}
\end{array}\right)\right\} \text {. }
$$

and $\Gamma^{\prime}$ is:

$$
\left\{\left(\begin{array}{c}
\frac{1}{\alpha_{0}} \\
0
\end{array}\right),\left(\begin{array}{c}
\frac{1}{2 \alpha_{0}} \\
b l
\end{array}\right)\right\}
$$

(notice that $\left.b^{2}=\frac{15}{16}\right)$.

Using the fact that 15 and 16 are coprimes we can parametrize $\left(\frac{\Gamma}{\Gamma^{\prime}}\right)$ as follows:

$$
\beta(x, y, s)=\left(\begin{array}{c}
0 \\
\frac{s}{2 b}
\end{array}\right)+\left(\begin{array}{c}
x \frac{\alpha_{0}}{2} \\
y b
\end{array}\right)
$$

with $x=0, \pm 1, \pm 2 \ldots k^{2}-4 ; y=0, \pm 1 \ldots l ; s=0, \pm 1 \ldots \pm 7$ and $x-y=$ even. Nothing changes in the expression (3.8) of $g_{1,2}^{ \pm}$: again $\mathrm{s}$ is invariant and only $x$ and $y$ are affected. The crucial difference with the previous analysis is in the structure of the group $\tilde{G}$.

First : this time, besides the usual symmetries $g_{u p}$ and $g_{\text {down }} \Gamma$ has also a $Z_{3}$ symmetry: we can write the weights of $\beta(x, y, s)$ as follows: (let us set $z=15 y+8 s$ for future convenience):

$$
h(x, z)=\frac{3\left(x^{2}-4\right)+z^{2}}{240}
$$

note that the lattice $\Gamma^{\prime}$ is described by

$$
\begin{aligned}
& x \rightarrow x+20 l_{1} \\
& z \rightarrow z+60 l_{2}
\end{aligned}
$$

with $l_{1}-l_{2}$ even. Then the transformations:

$$
\begin{aligned}
& x \rightarrow \frac{x \pm z}{2} \\
& z \rightarrow \frac{z \mp 3 x}{2}
\end{aligned}
$$


(notice that $x-z$ is even) have the following properties:

i] are weight preserving.

ii] commute with the lattice $\Gamma^{\prime}$

iii] are a realization of the $Z_{3}$ group.

Second: Notwithstanding the presence of a bigger group of weight preserving transformations the generators $g_{1,2}^{ \pm}$are not any more equivalent and the orbit under $\mathrm{G}$ will contain 6 elements instead of 2 . The orbit under the whole group $\tilde{G}$ will contain, depending on the characteristics of the field 36 or 72 elements.

The equivalence classes $\frac{\Gamma}{\Gamma^{\prime}}$ are organized by the action of $\tilde{G}$ on four independent fields: identity, $\epsilon$ of weight $\frac{2}{5} ; \sigma$ of weight $\frac{1}{15}$ and $\psi$ of weight $\frac{2}{3}$. We write explicitly the part generated by $G$ of their characters and omit the part generated by $G_{0}$ which is given by a straightforward application of $g_{u p}, g_{\text {down }}$ and of eqs. (3.24).

In the following $\left(\begin{array}{l}x \\ z\end{array}\right)$ will be a shorthand notation for:

$$
\left(\begin{array}{l}
x \\
z
\end{array}\right)=\sum_{l_{1}, l_{2} \in \mathbf{Z}} q^{h\left(x+20 l_{1}, z+60 l_{2}\right)}
$$

$l_{1}-l_{2}$ even. For each field we also give $N(\beta)$ and $n(\beta)$

A] Identity; $h=0, N(0)=36, n(0)=6$ :

$$
\chi_{0}=\left(\begin{array}{l}
2 \\
0
\end{array}\right)+\left(\begin{array}{l}
13 \\
15
\end{array}\right)+\left(\begin{array}{c}
13 \\
-15
\end{array}\right)-\left(\begin{array}{c}
18 \\
0
\end{array}\right)-\left(\begin{array}{c}
3 \\
15
\end{array}\right)-\left(\begin{array}{c}
3 \\
-15
\end{array}\right)
$$

B] $\epsilon$ field; $h=\frac{2}{5}, N=36, n=6$ :

$$
\chi_{\frac{2}{5}}=\left(\begin{array}{l}
6 \\
0
\end{array}\right)+\left(\begin{array}{c}
19 \\
15
\end{array}\right)+\left(\begin{array}{c}
19 \\
-15
\end{array}\right)-\left(\begin{array}{c}
14 \\
0
\end{array}\right)-\left(\begin{array}{c}
11 \\
15
\end{array}\right)-\left(\begin{array}{c}
11 \\
-15
\end{array}\right)
$$

C] $\sigma$ field; $h=\frac{1}{15}, N=72, n=12$ :

$$
\chi_{\frac{1}{15}}=\left(\begin{array}{c}
3 \\
1
\end{array}\right)+\left(\begin{array}{c}
3 \\
31
\end{array}\right)+\left(\begin{array}{c}
18 \\
16
\end{array}\right)-\left(\begin{array}{c}
13 \\
1
\end{array}\right)-\left(\begin{array}{c}
7 \\
-29
\end{array}\right)-\left(\begin{array}{c}
2 \\
16
\end{array}\right)
$$

D] $\psi$ field; $h=\frac{2}{3}, N=72, n=12$ :

$$
\chi_{\frac{2}{3}}=\left(\begin{array}{l}
6 \\
8
\end{array}\right)+\left(\begin{array}{c}
19 \\
7
\end{array}\right)+\left(\begin{array}{c}
1 \\
-37
\end{array}\right)-\left(\begin{array}{c}
14 \\
8
\end{array}\right)-\left(\begin{array}{c}
11 \\
7
\end{array}\right)-\left(\begin{array}{c}
9 \\
-37
\end{array}\right)
$$

The partition function is:

$$
\left|\chi_{0}\right|^{2}+\left|\chi_{\frac{2}{5}}\right|^{2}+2\left|\chi_{\frac{1}{15}}\right|^{2}+2\left|\chi_{\frac{2}{3}}\right|^{2}
$$


Which as expected is exactly the partition function of the three states Potts model. In Appendix $\mathrm{C}$ we show the equivalence between our characters $(3.25, \mathrm{a}, \mathrm{b}, \mathrm{c}, \mathrm{d})$ and the usual ones.

$$
\left.c=\frac{10}{7}\right]
$$

This model can be identified with the $\mathrm{p}=6$ case of the Fateev-Zamolodchikov series [11]. An hint for this identification is given not only by the correspondence of central charge and operator content, but also by the presence, as in the previous case of an additional $Z_{3}$ symmetry. In Appendix $\mathrm{C}$ we show the complete correspondence of our characters with the usual ones.

In this case we have to choose the set of screening charges (3.4) with $\mathrm{k}=5$ and $\mathrm{l}=2$. The analysis follows what we did for the $c=\frac{4}{5}$ model and we omit the details. This time the weight function is:

$$
h(x, z)=\frac{\left(x^{2}-4\right)+3 z^{2}}{168}
$$

where $z=7 y+4 s$ (notice again $x-z$ even) and the $Z_{3}$ symmetry is given by:

$$
\begin{aligned}
& x \rightarrow \frac{x \pm 3 z}{2} \\
& z \rightarrow \frac{z \mp x}{2}
\end{aligned}
$$

while the lattice $\Gamma^{\prime}$ is given by

$$
\begin{aligned}
& x \rightarrow x+42 l_{1} \\
& z \rightarrow z+14 l_{2}
\end{aligned}
$$

with $l_{1}-l_{2}$ even.

This time the elements of $\frac{\Gamma}{\Gamma^{\prime}}$ are organized in 8 fields. We give in the following the corresponding characters, what is new with respect to the previous model is that now $N(\beta)$ and $n(\beta)$ are non-trivial and this will induce a non-trivial partition function.

I] Identity: $h=0, N(0)=36, n(0)=6$ :

$$
\chi_{0}=\left(\begin{array}{l}
2 \\
0
\end{array}\right)+\left(\begin{array}{c}
19 \\
7
\end{array}\right)+\left(\begin{array}{c}
19 \\
-7
\end{array}\right)-\left(\begin{array}{c}
26 \\
0
\end{array}\right)-\left(\begin{array}{c}
5 \\
7
\end{array}\right)-\left(\begin{array}{c}
5 \\
-7
\end{array}\right)
$$

A] field of weight: $h=\frac{9}{14}, N=24, n=12$ :

$$
\chi_{\frac{9}{14}}=\left(\begin{array}{c}
10 \\
2
\end{array}\right)-\left(\begin{array}{c}
4 \\
-12
\end{array}\right)
$$

B] field of weight: $h=\frac{1}{14}, N=12, n=6$ :

$$
\chi_{\frac{1}{14}}=\left(\begin{array}{c}
4 \\
0
\end{array}\right)-\left(\begin{array}{c}
32 \\
0
\end{array}\right)
$$


C] field of weight: $h=\frac{5}{14}, N=12, n=6$ :

$$
\chi_{\frac{5}{14}}=\left(\begin{array}{c}
8 \\
0
\end{array}\right)-\left(\begin{array}{c}
20 \\
0
\end{array}\right)
$$

D] field of weight: $h=\frac{3}{2}, N=12, n=6$ :

$$
\chi_{\frac{3}{2}}=\left(\begin{array}{c}
16 \\
0
\end{array}\right)-\left(\begin{array}{c}
40 \\
0
\end{array}\right)
$$

E] field of weight: $h=\frac{1}{7}, N=72, n=12$ :

$$
\chi_{\frac{1}{7}}=\left(\begin{array}{l}
5 \\
1
\end{array}\right)+\left(\begin{array}{c}
5 \\
-13
\end{array}\right)+\left(\begin{array}{c}
26 \\
8
\end{array}\right)-\left(\begin{array}{l}
2 \\
8
\end{array}\right)-\left(\begin{array}{c}
19 \\
1
\end{array}\right)-\left(\begin{array}{c}
19 \\
3
\end{array}\right)
$$

F] field of weight: $h=\frac{4}{7}, N=36, n=6$ :

$$
\chi_{\frac{4}{7}}=\left(\begin{array}{c}
10 \\
0
\end{array}\right)+\left(\begin{array}{c}
11 \\
7
\end{array}\right)+\left(\begin{array}{c}
11 \\
-7
\end{array}\right)-\left(\begin{array}{c}
4 \\
14
\end{array}\right)-\left(\begin{array}{c}
17 \\
7
\end{array}\right)-\left(\begin{array}{c}
17 \\
-7
\end{array}\right)
$$

G] field of weight: $h=\frac{6}{7}, N=36, n=6$ :

$$
\chi_{\frac{6}{7}}=\left(\begin{array}{c}
10 \\
4
\end{array}\right)+\left(\begin{array}{c}
11 \\
-3
\end{array}\right)+\left(\begin{array}{c}
11 \\
11
\end{array}\right)-\left(\begin{array}{c}
4 \\
-10
\end{array}\right)-\left(\begin{array}{c}
17 \\
11
\end{array}\right)-\left(\begin{array}{c}
17 \\
-3
\end{array}\right)
$$

The partition function is

$$
\left|\chi_{0}\right|^{2}+6\left|\chi_{\frac{9}{14}}\right|^{2}+3\left|\chi_{\frac{1}{14}}\right|^{2}+3\left|\chi_{\frac{5}{14}}\right|^{2}+3\left|\chi_{\frac{3}{2}}\right|^{2}+2\left|\chi_{\frac{1}{7}}\right|^{2}+\left|\chi_{\frac{4}{7}}\right|^{2}+\left|\chi_{\frac{6}{7}}\right|^{2}
$$

which is exactly the modular invariant of the complementary series $[20,22]$ at level $p=6$ of the $\frac{S U(3) \times S U(3)}{S U(3)}$ models.

In Appendix B we show the equivalence between these characters and the corresponding $\frac{S U(3) \times S U(3)}{S U(3)}$ characters.

E] As a final example we describe a model with an asymmetric choice of the screening charges:

Let us take:

$$
\alpha_{0}=\left(\begin{array}{c}
\frac{2}{\sqrt{15}} \\
0
\end{array}\right)
$$

then $c=2-24 \alpha_{0}=-\frac{22}{5}$.

Let us choose the charges as:

$$
e_{1}^{+}=\frac{1}{4}\left(\begin{array}{c}
5 \sqrt{\frac{5}{3}} \\
1
\end{array}\right), \quad e_{1}^{-}=\frac{1}{4}\left(\begin{array}{c}
-3 \sqrt{\frac{3}{5}} \\
-1
\end{array}\right)
$$




$$
e_{2}^{+}=\frac{1}{4}\left(\begin{array}{c}
-\sqrt{\frac{5}{3}} \\
3
\end{array}\right), \quad e_{2}^{-}=\frac{1}{4}\left(\begin{array}{c}
7 \sqrt{\frac{3}{5}} \\
-3
\end{array}\right)
$$

with the usual analysis we obtain:*

$$
\Gamma=\left\{\left(\begin{array}{l}
0 \\
2
\end{array}\right),\left(\begin{array}{c}
\frac{1}{4 \sqrt{15}} \\
\frac{1}{4}
\end{array}\right)\right\} .
$$

and then:

$$
\Gamma^{\prime}=\left\{\left(\begin{array}{c}
-\frac{\sqrt{1} 5}{4} \\
\frac{1}{4}
\end{array}\right),\left(\begin{array}{c}
2 \sqrt{15} \\
0
\end{array}\right)\right\}
$$

The weight of an element $\left(\begin{array}{c}\frac{x}{4 \sqrt{15}} \\ \frac{y}{4}\end{array}\right)$ of $\Gamma$ is

$$
h(x, y)=\frac{x^{2}+15 y^{2}-64}{240}
$$

The only weight preserving transformation allowed in $\Gamma$ is $:(x, y) \rightarrow(-x,-y)$. The matrices $g_{1,2}^{ \pm}$are:

$$
\begin{aligned}
g_{1}^{ \pm} & = \pm \frac{1}{8}\left(\begin{array}{cc}
17 & -225 \\
1 & -17
\end{array}\right) \\
g_{2}^{ \pm} & = \pm \frac{1}{8}\left(\begin{array}{cc}
-13 & 45 \\
3 & -35
\end{array}\right)
\end{aligned}
$$

and one can show that all the $g_{1,2}^{ \pm}$'s are equivalent. The action of $\tilde{G}$ on $\frac{\Gamma}{\Gamma^{\prime}}$ leave us with the following 2 characters: let us set:

$$
\left(\begin{array}{l}
x \\
y
\end{array}\right)=\sum_{l_{1}, l_{2}} q^{h\left(x+15 l_{1}, y-l_{1}+8 l_{2}\right)}
$$

we have:

- Identity: $h=0$

$$
\left(\begin{array}{l}
8 \\
0
\end{array}\right)-\left(\begin{array}{l}
2 \\
2
\end{array}\right)
$$

- $\phi$ field: $h=-\frac{1}{5}$

$$
\left(\begin{array}{l}
1 \\
1
\end{array}\right)-\left(\begin{array}{l}
4 \\
4
\end{array}\right)
$$

we made a computer check up to $q^{100}$ and found that these are exactly the well known Virasoro characters for the $c=-22 / 5$ model.

* Notice that in this case there is another consistent choice for $\Gamma$, which gives, due to non-trivial lattice identities the same results as this one. 


\section{Section 4: Examples: correlation functions}

Note that in this section we come back to the original definition of the lattice $\Gamma$ (unshifted $\Gamma$ ). To make contact between the states $\beta$ of sect. 3 and the one we will use in this section one must add $\alpha_{0}$ to the values of $\beta$ of sect. 3

A] The $c=-\frac{22}{5}$ model

In this case there is only one non trivial 4-pt. correlation function in which all the fields have weight $-\frac{1}{5}$. Let us choose as representative of this field our state:

$$
\beta=\left(\begin{array}{c}
\frac{9}{4 \sqrt{15}} \\
\frac{1}{4}
\end{array}\right)
$$

which can be screened by using two screening charges of type $e_{1}^{-}$(the other choice: $\beta=$ $\left(\begin{array}{c}\frac{7}{4 \sqrt{15}} \\ \frac{1}{4}\end{array}\right)$, as usual, would require a more complicate combination of screenings)

We have:

$$
\begin{gathered}
<\Phi_{-\frac{1}{5}}(0) \Phi_{-\frac{1}{5}}(z) \Phi_{-\frac{1}{5}}(1) \Phi_{-\frac{1}{5}}(\infty)> \\
=\oint d w_{1} d w_{2}<V_{\beta}(0) V_{\beta}(z) V_{\beta}(1) V_{2 \alpha_{0}-\beta}(\infty) V_{e_{1}^{-}}\left(w_{1}\right) V_{e_{1}^{-}}\left(w_{2}\right)
\end{gathered}
$$

namely:

$$
\begin{gathered}
z^{2 \beta^{2}}(1-z)^{2 \beta^{2}} \oint d w_{1} d w_{2} w_{1}^{2 \beta e_{1}^{-}} w_{2}^{2 \beta e_{1}^{-}}\left(1-w_{1}\right)^{2 \beta e_{1}^{-}}\left(1-w_{2}\right)^{2 \beta e_{1}^{-}} \\
\left(z-w_{1}\right)^{2 \beta e_{1}^{-}}\left(z-w_{2}\right)^{2 \beta e_{1}^{-}}\left(w_{1}-w_{2}\right)^{2\left(e_{1}^{-}\right)^{2}} \\
=z^{\frac{4}{5}}(1-z)^{\frac{4}{5}} \oint d w_{1} d w_{2} w_{1}^{-\frac{4}{5}} w_{2}^{-\frac{4}{5}}\left(1-w_{1}\right)^{-\frac{4}{5}}\left(1-w_{2}\right)^{-\frac{4}{5}}\left(z-w_{1}\right)^{-\frac{4}{5}}\left(z-w_{2}\right)^{-\frac{4}{5}}\left(w_{1}-w_{2}\right)^{\frac{4}{5}}
\end{gathered}
$$

Let us look now at the model in the context of the Virasoro series: it is given by $\mathrm{p}=2$, $\mathrm{p}^{\prime}=5$ and the field of weight $h=-\frac{1}{5}$ is described by $\beta_{1}=-\frac{\alpha_{-}}{2}$ or $\beta_{2}=-\alpha_{-}$(note again the conformal grid symmetry) with $\alpha_{-}=-\sqrt{\frac{2}{5}}$. Let us take $\beta_{2}$ and let us screen it using 2 $\alpha_{-}$charges. Due to the fact that $2 \beta_{2}^{2}=\frac{4}{5}, 2 \alpha_{-}^{2}=\frac{4}{5}$ and $2 \alpha_{-} \beta_{2}=-\frac{4}{5}$ the two integrands are completely equivalent.

B] Let us look now at a less trivial case: the Ising model which is in the

$$
\frac{S U^{p}(2) \times S U^{1}(2) \times S U^{1}(2)}{S U^{p+2}(2)}
$$

series at the level $\mathrm{n}=1$ which in fact correspond to $\mathrm{p}=0$. 
The screening charges are:

$$
e_{1,2}^{+}=\left(\begin{array}{c}
1 \\
\pm \frac{1}{\sqrt{2}}
\end{array}\right), \quad \quad e_{1,2}^{-}=\left(\begin{array}{c}
-\frac{1}{2} \\
\pm \frac{1}{\sqrt{2}}
\end{array}\right)
$$

a good representative for the $\sigma$ field is

$$
\sigma=\left(\begin{array}{c}
\frac{1}{4} \\
\frac{1}{2 \sqrt{2}}
\end{array}\right)
$$

which can be screened by using one $e_{2}^{-}$screening charge.

Looking at the $\epsilon$ field we see that there are three contributions at the same level. Following the general prescription of sect. 2 we know that all of them must be taken into account: we choose as representatives for $\epsilon$ :

$$
\epsilon=\left(\begin{array}{c}
0 \\
\frac{1}{\sqrt{2}}
\end{array}\right)+\left(\begin{array}{c}
0 \\
-\frac{1}{\sqrt{2}}
\end{array}\right)-\left(\begin{array}{l}
1 \\
0
\end{array}\right) .
$$

The correlation function $\langle\sigma \sigma \sigma \sigma\rangle$ is very simple to handle and gives directly the known result. Let us study the less trivial case; $\langle\epsilon \sigma \sigma \epsilon>$

We have in general:

$$
\begin{aligned}
& \oint d w<V_{\epsilon}(0) V_{\sigma}(z) V_{\sigma}(1) V_{2 \alpha_{0}-\epsilon}(\infty) V_{e_{2}^{-}}(w)> \\
= & z^{2 \epsilon \sigma}(1-z)^{2 \sigma^{2}} \oint d w w^{2 \epsilon e_{2}^{-}}(1-w)^{2 \sigma e_{2}^{-}}(z-w)^{2 \sigma e_{2}^{-}} \\
= & z^{2 \epsilon \sigma}(1-z)^{\frac{3}{8}} \oint d w w^{2 \epsilon e_{2}^{-}}(1-w)^{-\frac{3}{4}}(z-w)^{-\frac{3}{4}}
\end{aligned}
$$

Let us take first $\epsilon=\left(\begin{array}{c}0 \\ \frac{1}{\sqrt{2}}\end{array}\right)$, then $2 \sigma \epsilon=\frac{1}{2}$ and $2 \epsilon e_{2}^{-}=-1$. Since the power of $w$ is -1 there are no branches in $w=0$ and we must choose (see below for some remarks on this point) the contour from 1 to $\infty$ (we have only one intermediate state: $\sigma$ itself). Let us transform $w \rightarrow \frac{1}{w}$, then:

$$
\begin{gathered}
z^{\frac{1}{2}}(1-z)^{\frac{3}{8}} \int_{0}^{1} d w w^{\frac{1}{2}}(1-w)^{-\frac{3}{4}}(1-w z)^{-\frac{3}{4}} \\
=z^{\frac{1}{2}}(1-z)^{\frac{3}{8}} \frac{\Gamma\left(\frac{3}{2}\right) \Gamma\left(\frac{1}{4}\right)}{\Gamma\left(\frac{7}{4}\right)} F\left(\frac{3}{4}, \frac{3}{2}, \frac{7}{4} ; z\right)
\end{gathered}
$$

Similar manipulations allow us to write the other two cases: if $\epsilon=\left(\begin{array}{c}0 \\ -\frac{1}{\sqrt{2}}\end{array}\right)$, then $2 \sigma \epsilon=-\frac{1}{2}$ and $2 \epsilon e_{2}^{-}=1$ and we end up with:

$$
=z^{-\frac{1}{2}}(1-z)^{\frac{3}{8}} \frac{\Gamma\left(-\frac{1}{2}\right) \Gamma\left(\frac{1}{4}\right)}{\Gamma\left(-\frac{1}{4}\right)} F\left(\frac{3}{4},-\frac{1}{2},-\frac{1}{4} ; z\right)
$$


Finally taking $\epsilon=\left(\begin{array}{l}1 \\ 0\end{array}\right)$, we exactly find $(4.1, a)$. Since $\left(\begin{array}{l}1 \\ 0\end{array}\right)$ appears in the character with a minus sign the two cancel exactly and we end up with $(4.1, \mathrm{~b})$ as the final result which is indeed the right answer [1]. Notice as a final remark that in $(4.1, a)$ we could also choose in principle a contour winding around the simple pole in $\mathrm{w}=0$. This would give a contribution $z^{-\frac{1}{4}}(1-z)^{\frac{3}{4}}$ which cannot exist since by power counting it would imply the propagation of an intermediate state of weight $\frac{5}{16}$ which do not exist. In fact this term is cancelled by the corresponding one due to the $\left(\begin{array}{l}1 \\ 0\end{array}\right)$ field. The presence of such spurious states is a common feature of most of the models that we describe and in all the cases that we have examinated they exactly cancel.

C] As a final example we discuss the correlation functions for the first model in the spin $\frac{4}{3}$ parafermionic series [18] , namely the $c=\frac{6}{7}$ model. Here, as pointed out earlier, dimension $\frac{4}{3}$ field appears in a very non-trivial way. Starting from $\beta=\left(\begin{array}{c}0 \\ \frac{2}{3}\end{array}\right)$ which has dimension $\frac{1}{3}$, the character $\chi_{\beta}$ exhibits a surprising feature: $g \beta$ which gives the "Weyl" action on $\beta$ happens to be: $\left(\begin{array}{c}-3 \\ \frac{1}{3}\end{array}\right)$ which also has dimension $\frac{1}{3}$. As a result dimension $\frac{1}{3}$ field disappears from the spectrum as it should and one begins with dimension $\frac{4}{3}$ field. We will here evaluate the correlation function involving this dimension $\frac{4}{3}$ field. This involves starting from the torus correlation function first order expansion in powers of $q$ and thereby provide a very non trivial check for our prescription for correlation functions. But before doing so, first we shall show that the dimension $\frac{1}{3}$ field which disappears from the spectrum also decouples from the correlation functions.

Consider the correlation function $\mathrm{G}(\mathrm{z})$ with two dimension $\frac{1}{3}$ fields and two $\frac{10}{21}$ fields. The field $\frac{10}{21}$ is given by: $\left(\begin{array}{c}-2 \\ \pm \frac{2}{3}\end{array}\right)$. Following the prescription given in sect.2 eq.(2.16) we have:

$$
G(z)=\int_{1}^{\infty} \int_{1}^{\infty} d w_{1} d w_{2} I_{1}\left(w_{1}, w_{2}, z\right)-\int_{1}^{\infty} d w_{1} \int_{0}^{z} d w_{2} I_{2}\left(w_{1}, w_{2}, z\right)
$$

where:

$$
\begin{aligned}
I_{1}=\sum_{\epsilon= \pm 1} I_{1}^{(\epsilon)}= & \sum_{\epsilon= \pm 1}<V\left(\begin{array}{c}
0 \\
\epsilon \frac{2}{3}
\end{array}\right)^{(0) V}\left(\begin{array}{c}
-2 \\
\epsilon \frac{2}{3}
\end{array}\right)^{(z) V}\left(\begin{array}{c}
-2 \\
-\epsilon \frac{2}{3}
\end{array}\right) \\
& V\left(\begin{array}{c}
4 \\
-\epsilon \frac{2}{3}
\end{array}\right)^{(\infty) V}\left(\begin{array}{l}
7 \\
1
\end{array}\right)^{\left(w_{1}\right) V}\left(\begin{array}{l}
-3 \\
-1
\end{array}\right)\left(w_{2}\right)>
\end{aligned}
$$


and

$$
\begin{aligned}
& I_{2}=\sum_{\epsilon= \pm 1} I_{2}^{(\epsilon)}=\sum_{\epsilon= \pm 1}<V\left(\begin{array}{c}
-3 \\
-\epsilon \frac{1}{3}
\end{array}\right)^{(0) V}\left(\begin{array}{c}
-2 \\
\epsilon \frac{2}{3}
\end{array}\right)^{(z) V}\left(\begin{array}{c}
-2 \\
-\epsilon \frac{2}{3}
\end{array}\right)^{(1)} \\
& V\left(\begin{array}{c}
7 \\
\epsilon \frac{1}{3}
\end{array}\right)^{(\infty) V}\left(\begin{array}{l}
7 \\
1
\end{array}\right)^{\left(w_{1}\right) V}\left(\begin{array}{l}
-3 \\
-1
\end{array}\right)\left(w_{2}\right)>
\end{aligned}
$$

Note that in the $I_{2}$ integral $w_{2}$ contour flips from $\int_{1}^{\infty}$ to $\int_{0}^{z}$ according to prescription given in sect.2. These integrals correspond to the s-channel with intermediate block being that of dimension $\frac{10}{21}$ field. Actually by a simple power counting it is clear that both the integrals start from the first secondary of $\frac{10}{21}$ field. To the lowest power in z (namely $z^{\frac{2}{3}}$ ) both the terms in $I_{1}$ and only one term ; $\epsilon=1$ in $I_{2}$ contribute.

$$
\begin{gathered}
I_{1}=\int_{1}^{\infty} d w_{1} \int_{1}^{\infty} d w_{2} w_{1}^{\frac{1}{3}}\left(w_{1}-1\right)^{-\frac{1}{3}} w_{2}^{-\frac{1}{7}}\left(w_{2}-1\right)^{\frac{1}{7}}\left[\frac{w_{2}^{2}\left(w_{1}-1\right)}{w_{1}^{2}\left(w_{2}-1\right)}+\frac{w_{1}^{2}\left(w_{2}-1\right)}{w_{2}^{2}\left(w_{1}-1\right)}\right]\left(w_{1}-w_{2}\right)^{-2} \\
I_{2}=\int_{1}^{\infty} d w_{1} \int_{0}^{1} d w_{2} w_{1}^{-\frac{7}{3}}\left(w_{1}-1\right)^{-\frac{4}{3}} w_{2}^{\frac{5}{7}}\left(1-w_{2}\right)^{-\frac{6}{7}}
\end{gathered}
$$

where in $I_{2}$ we have scaled $w_{2} \rightarrow z w_{2} . I_{2}$ gives two decoupled integrals and the result is:

$$
I_{2}=\frac{\Gamma\left(\frac{8}{3}\right) \Gamma\left(-\frac{1}{3}\right)}{\Gamma\left(\frac{7}{3}\right)} \frac{\Gamma\left(\frac{12}{7}\right) \Gamma\left(\frac{1}{7}\right)}{\Gamma\left(\frac{13}{7}\right)}
$$

To calculate $I_{1}$, we change variables: $w_{1} \rightarrow \frac{1}{w_{1}}$ and $w_{2} \rightarrow \frac{1}{w_{2}}$ :

$$
I_{1}=\int_{0}^{1} d w_{1} \int_{0}^{1} d w_{2} w_{1}^{\frac{1}{3}}\left(1-w_{1}\right)^{-\frac{1}{3}} w_{2}^{-\frac{1}{7}}\left(1-w_{2}\right)^{\frac{1}{7}}\left[\frac{w_{1}\left(1-w_{1}\right)}{w_{2}\left(1-w_{2}\right)}+\frac{w_{2}\left(1-w_{2}\right)}{w_{1}\left(1-w_{1}\right)}\right]\left(w_{1}-w_{2}\right)^{-2}
$$

Further we note that:

$$
\left[\frac{A}{B}+\frac{B}{A}\right]=\frac{(A-B)^{2}}{A B}+2
$$

Using this:

$$
\left[\frac{w_{1}\left(1-w_{1}\right)}{w_{2}\left(1-w_{2}\right)}+\frac{w_{2}\left(1-w_{2}\right)}{w_{1}\left(1-w_{1}\right)}\right]=\left(w_{1}-w_{2}\right)^{2} \frac{\left[1-w_{1}-w_{2}\right]^{2}}{w_{1} w_{2}}+2
$$

The first term gives two decoupled integrals while the second one:

$$
2 \int_{0}^{1} d w_{1} \int_{0}^{1} d w_{2} w_{1}^{\frac{1}{3}}\left(1-w_{1}\right)^{-\frac{1}{3}} w_{2}^{-\frac{1}{7}}\left(1-w_{2}\right)^{\frac{1}{7}}\left(w_{1}-w_{2}\right)^{-2}
$$

is exactly of the type discussed in Appendix D and can be evaluated using eqs.(D1-4) . By regrouping terms one can see that $I_{1}=I_{2}$ and therefore to this order in $\mathrm{z}, \frac{1}{3}$ field decouples. Although we have not proven this to all orders in $\mathrm{z}$, we believe the same feature will persist. 
Now let us go to the $\frac{4}{3}$ field. From the torus 2-pt. function we must extract the next to leading power in $q$ namely $q^{\frac{4}{3}-\frac{c}{24}}$. At this order of $q$, several terms contribute: firstly we must expand $\theta$-functions appearing in eq.(2.14). The expansion of $\theta$-functions is as follows:

$$
\theta(x-y) \rightarrow q^{\frac{1}{8}} \frac{x-y}{\sqrt{x y}}\left[1-q\left(\frac{x}{y}+\frac{y}{x}+1\right) \cdots\right]
$$

in the limit in which: $q \rightarrow 0, x \rightarrow \frac{\ln x}{2 \pi i}, y \rightarrow \frac{\ln y}{2 \pi i}$. We then have to multiply the integrands $I_{1,2}^{\epsilon}$ by the corresponding factors inside the bracket in eq.(4.8). It is easy to see that these terms again contribute to the first secondary of the $\frac{10}{21}$ fields.

Secondly to the next order in $q$, there is also a term coming from the lattice namely $\left(\begin{array}{c}0 \\ \pm \frac{4}{3}\end{array}\right)$. Thus we have :

$$
\begin{gathered}
I_{3}=\sum_{\epsilon= \pm 1} I_{3}^{(\epsilon)}=\sum_{\epsilon= \pm 1}<V_{\left(\begin{array}{c}
0 \\
-\epsilon \frac{4}{3}
\end{array}\right)^{(0) V}\left(\begin{array}{c}
-2 \\
\epsilon \frac{2}{3}
\end{array}\right)^{(z) V}\left(\begin{array}{c}
-2 \\
-\epsilon \frac{2}{3}
\end{array}\right)}^{(1)} \\
V_{\left(\begin{array}{c}
4 \\
\epsilon \frac{2}{3}
\end{array}\right)^{(\infty) V}\left(\begin{array}{l}
7 \\
1
\end{array}\right)^{\left(w_{1}\right) V}\left(\begin{array}{l}
-3 \\
-1
\end{array}\right)}^{\left(w_{2}\right)>}
\end{gathered}
$$

Again by power counting one can see that this time the intermediate state starts from the primary i.e. at level $\frac{10}{21}$. The integrals this time are

$$
\begin{aligned}
I_{3}^{(\epsilon)}=z^{-\frac{4}{3}}(1-z)^{-\frac{4}{7}} & \int_{1}^{\infty} d w_{1} \int_{1}^{\infty} d w_{2}\left(w_{1}-1\right)^{-\frac{1}{3}}\left(w_{2}-1\right)^{\frac{1}{7}}\left(w_{1}-z\right)^{-\frac{1}{3}}\left(w_{2}-z\right)^{\frac{1}{7}} \\
& {\left[\frac{w_{1}^{2}\left(w_{2}-z\right)\left(w_{1}-1\right)}{w_{2}^{2}\left(w_{1}-z\right)\left(w_{2}-1\right)}\right]^{\epsilon}\left(w_{1}-w_{2}\right)^{-2} }
\end{aligned}
$$

Changing the variables $w_{1} \rightarrow \frac{1}{w_{1}}$ and $w_{2} \rightarrow \frac{1}{w_{2}}$ and to the lowest order in $\mathrm{z}$ we get:

$$
I_{3}^{(\epsilon)}(0)=\int_{0}^{1} d w_{1} \int_{0}^{1} d w_{2} w_{1}^{\frac{2}{3}}\left(1-w_{1}\right)^{-\frac{1}{3}} w_{2}^{-\frac{2}{7}}\left(1-w_{2}\right)^{\frac{1}{7}}\left[\frac{w_{2}^{2}\left(1-w_{1}\right)}{w_{1}^{2}\left(1-w_{2}\right)}\right]^{\epsilon}\left(w_{1}-w_{2}\right)^{-2}
$$

Once again using methods similar to the one leading to eq.(4.7) we can write $I_{3}^{+}+I_{3}^{-}$in terms of two terms: one which is of the form as in eq. (4.7) and the second decouples. The result is:

$$
I_{3}(0)=z^{-\frac{4}{3}}\left[-\frac{50}{9} N\left(\frac{2}{3},-\frac{1}{3},-\frac{2}{7}, \frac{1}{7}\right)+O(z)\right]
$$

(see appendix $\mathrm{D}$ for the definition of $N(a, b, c, d))$ Now let us go to the next order in $\mathrm{z}$. There are several terms now: Firstly the ones coming from the expansion of $I_{3}^{(\epsilon)}$, and secondly the leading terms coming from $I_{1}$ and $I_{2}$ multiplied by the $\theta$-function expansions. The terms coming from the expansion of $I_{3}^{(\epsilon)}$ are:

$$
z^{-\frac{1}{3}}\left(\frac{4}{7} I_{3}(0)+\sum_{\epsilon} I_{3}^{(\epsilon)}(1)\right)
$$


where:

$$
\begin{aligned}
I_{3}^{(\epsilon)}(1)= & \int_{0}^{1} d w_{1} \int_{0}^{1} d w_{2} w_{1}^{\frac{2}{3}}\left(1-w_{1}\right)^{-\frac{1}{3}} w_{2}^{-\frac{2}{7}}\left(1-w_{2}\right)^{\frac{1}{7}}\left[\frac{w_{2}^{2}\left(1-w_{1}\right)}{w_{1}^{2}\left(1-w_{2}\right)}\right]^{\epsilon} \\
& \left(w_{1}-w_{2}\right)^{-2}\left[\frac{1}{3} w_{1}-\frac{1}{7} w_{2}+\epsilon\left(w_{1}-w_{2}\right)\right]
\end{aligned}
$$

Now using the following relation:

$$
\begin{aligned}
& {\left[\frac{w_{2}^{2}\left(1-w_{1}\right)}{w_{1}^{2}\left(1-w_{2}\right)}\right]^{\epsilon}\left[\frac{1}{3} w_{1}-\frac{1}{7} w_{2}+\epsilon\left(w_{1}-w_{2}\right)\right]} \\
& =\left[\frac{\left(w_{1}-w_{2}\right)^{2}\left(w_{1}+w_{2}-w_{1} w_{2}\right)^{2}}{w_{1}^{2}\left(1-w_{1}\right) w_{2}^{2}\left(1-w_{1}\right)}+2\right]\left(\frac{1}{3} w_{1}-\frac{1}{7} w_{2}\right)+\frac{\left(w_{1}-w_{2}\right)^{2}\left[-w_{1}-w_{2}+w_{1} w_{2}\right]}{w_{1}^{2}\left(1-w_{1}\right) w_{2}^{2}\left(1-w_{1}\right)}
\end{aligned}
$$

The integral again splits into one which is decoupled and the other of the form of eq.(D.5). The result is:

$$
\sum_{\epsilon} I_{3}^{(\epsilon)}(1)=z^{-\frac{1}{3}}\left[\frac{776}{189} N\left(\frac{2}{3},-\frac{1}{3},-\frac{2}{7}, \frac{1}{7}\right)+O(z)\right] .
$$

Note that each of $I_{3}^{(\epsilon)}(1)$ satisfy the condition $\frac{a}{a^{\prime}}=\frac{b}{b^{\prime}}$ (see appendix D) and hence is of the form of eq.(D.1). For example:

$$
I_{3}^{(+1)}(1)=\int_{0}^{1} d w_{1} \int_{0}^{1} d w_{2} w_{1}^{-\frac{4}{3}}\left(1-w_{1}\right)^{\frac{2}{3}} w_{2}^{\frac{12}{7}}\left(1-w_{2}\right)^{-\frac{6}{7}}\left(w_{1}-w_{2}\right)^{-2}\left[\frac{4}{3} w_{1}-\frac{8}{7} w_{2}\right]
$$

but if we had used the formula $\left\langle w_{1}\right\rangle=<w_{2}>=\frac{a}{a+b}$ for $I_{3}^{( \pm 1)}$ separately we would not get the answer (4.15). The reason is that $\left.<\frac{4}{3} w_{1}-\frac{8}{7} w_{2}\right\rangle$ is not of the form $\left\langle a w_{1}+a^{\prime} w_{2}\right\rangle$ and as pointed out in the appendix the correction terms in the formula $\left.\left\langle w_{1}\right\rangle=<w_{2}\right\rangle=\frac{a}{a+b}$ drop out only in the latter combination.

Now let us include the terms coming from $I_{1}^{(\epsilon)}$ after the expansion of $\theta$-functions. To this order in $\mathrm{z}$ the only terms that contribute are:

$$
\begin{array}{r}
I_{1}^{(\epsilon)}(1)=z^{-\frac{1}{3}} \int_{0}^{1} d w_{1} \int_{0}^{1} d w_{2} w_{1}^{\frac{2}{3}}\left(1-w_{1}\right)^{-\frac{1}{3}} w_{2}^{-\frac{2}{7}}\left(1-w_{2}\right)^{\frac{1}{7}}\left[\frac{w_{1}\left(1-w_{1}\right)}{w_{2}\left(1-w_{2}\right)}\right]^{\epsilon} \\
\left(w_{1}-w_{2}\right)^{-2}\left[\frac{1}{3 w_{1}}-\frac{1}{7 w_{2}}+\epsilon\left(\frac{1}{w_{1}}-\frac{1}{w_{2}}\right)\right] .
\end{array}
$$

Once again going through the procedure of eq.(4.8) one can reduce this integral $\sum_{\epsilon} I_{1}^{(\epsilon)}(1)$ to a piece which is decoupled and another of the form (4.8). The result is (use eq.(D.9) of Appendix D):

$$
\sum_{\epsilon} I_{1}^{(\epsilon)}(1)=z^{-\frac{1}{3}}\left[-\frac{40}{21} N\left(\frac{2}{3},-\frac{1}{3},-\frac{2}{7}, \frac{1}{7}\right)\right] .
$$


Finally we have the terms coming from $I_{2}$ integral after expanding $\theta$-functions. Expanding $\theta$-functions to the first order in q, scaling $w_{2} \rightarrow z w_{2}, w_{1} \rightarrow \frac{1}{w_{1}}$ and extracting the terms of the order $z^{-\frac{1}{3}}$ we obtain:

$$
I_{2}(1)=z^{-\frac{1}{3}} \int_{0}^{1} d w_{1} \int_{0}^{1} d w_{2} w_{1}^{\frac{5}{3}}\left(1-w_{1}\right)^{-\frac{4}{3}} w_{2}^{\frac{5}{7}}\left(1-w_{2}\right)^{-\frac{6}{7}}\left[-\frac{2}{3 w_{1}}-\frac{8}{7 w_{2}}+\frac{2}{w_{1} w_{2}}\right]
$$

These are decoupled integrals and the result is:

$$
I_{2}(1)=z^{-\frac{1}{3}}\left[-\frac{8}{63} N\left(\frac{2}{3},-\frac{1}{3},-\frac{2}{7}, \frac{1}{7}\right)\right] .
$$

Adding $I_{1}(1)$ and $I_{3}(1)$ and subtracting $I_{2}(1)$ (as the latter comes from the negative part of the lattice) we obtain finally the correlation function as:

$$
G(z)=z^{-\frac{4}{3}}\left(-\frac{50}{9}\right) N\left(\frac{2}{3},-\frac{1}{3},-\frac{2}{7}, \frac{1}{7}\right)\left[1+\frac{16}{105} z+O\left(z^{2}\right)+\cdots\right]
$$

We have checked that this is in fact, upto an overall normalization, the correct expression of the corresponding correlator evaluated in the Virasoro model using Dotsenko-Fateev (1boson) approach [9].

We would like to emphasize that this example involved the expansion of 2-pt. function on the torus to next to leading order in $q$. Thus this is indeed a very non trivial check of our prescription for computing correlation functions. We have only checked upto the first order in $z$. Explicit calculations for higher orders in $z$ are considerably more cumbersome, however one may try to show the correctness of our correlation function by obtaining the differential equation it satisfies and comparing with the correct differential equation.

As a final remark we stress that we made checks upto the first order in $z$ for several other 4-pt. correlation functions of this model (in particular in the cases in which the screened fields are those of weight $h=\frac{1}{7}$ and $h=\frac{10}{21}$ ) and we have always found a perfect agreement with the corresponding functions evaluated in the 1 boson approach. 


\section{Section 5: Conclusions}

In this paper we have proposed a new approach to constructing RCFT's whose characters are given by lattice sums. We have also proposed a method to construct correlation functions for these models. In particular we have studied the case of 2-bosons systems. As an examples we have obtained a bosonic realization of spin $\frac{4}{3}$ parafermionic series, which allows one to construct torus correlation functions for these models.

There are several open problems one can study. Firstly one can address the question of completeness of GKO construction for unitary RCFT's. Another question is that of constructing new non-unitary series. It may also be interesting to extend the above analysis to the case of higher number of bosons which may be useful in building the classical solutions of string theory. In particular for 3-bosons case one should be able to obtain $\mathrm{N}=2$ Superconformal series. At a more conceptual level one would like to understand the generalization of Feigin-Fuchs procedure for these models. Similarly, although the general prescriptions for correlation functions we have given here works for the examples we have studied one would like to understand them better.

In particular one would like to understand to what extent different choices of screening give the same result. Even in the one boson case [9] the results are not free from this ambiguity for 5-pts. functions and more. In the present paper we have given some conditions in the screening choice which are implied by the requirement of well defined monodromy properties on the torus. It would be important to obtain a complete set of such conditions. Finally in order to construct more non trivial examples it is necessary to understand better the nature of the "Weyl" group action.

\section{Acknowledgements}

We would like to thank the following persons for several useful and stimulating discussions: V.Fateev, P.Fre', F.Gliozzi, T.Jayaraman, F. Ravanini and G.Sotkov. We acknowledge Prof. A.Salam, Director, ICTP,IAEA and UNESCO for supporting our work at the International Center for Theoretical Physics, Trieste. 
[1] A.A.Belavin, A.M.Polyakov and A.B.Zamolodchikov, Nucl. Phys. B241 (1984) 333

[2] D.Gepner, Nucl.Phys. B296 (1988) 757

[3] T.Eguchi and A.Taormina, CERN-TH 5123/88

[4] E.Verlinde, Nucl. Phys. B300 (1988) 360.

[5] C.Vafa, Phys. Lett. 206B (1988) 421

[6] S.D.Mathur, S.Mukhi and A.Sen, TIFR/TH/88-32.

[7] S.D.Mathur, S.Mukhi and A.Sen, TIFR/TH/88-50.

[8] P.Christe and F.Ravanini, NORDITA preprint 88/35P

[9] V.S.Dotsenko and V.Fateev, Nucl. Phys. B240 (1984) 312 and Nucl. Phys. B251 (1985) 691.

[10] B.L.Feigin and D.B.Fuchs, Funkts. Anal. Pril. 16 (1982) 47

[11] V.A.Fateev and A.B.Zamolodchikov, Nucl. Phys. B280 [FS18] (1987) 644

[12] V.A.Fateev and S.L.Lykyanov, Int. Journ. Mod. Phys. A3 (1988) 507

[13] P.Goddard, A.Kent, D.Olive, Comm. Math. Phys. 103 (1986) 105

[14] R.Dijkgraaf and E.Verlinde, THU-88/25

[15] T.Jayaraman and K.S.Narain, CERN-TH 5166/88 (IC/88/306);

G.Felder, ETH Preprint (1988); Al.Zamolodchikov, ITEP preprint (1988);

C.Crnkovic, G.Sotkov and M.Stanishkov, ICTP preprint (1988);

O.Foda and B.Nienhuis preprint (1988)

[16] J.Bagger, D.Nemeschansky and J.B.Zuber HUTP-88/A051 (USC-88/009)

[17] V.A.Fateev, private communication

[18] V.A.Fateev and A.B.Zamolodchikov, Teor. Math. Phys. 71 (1987) 451

[19] J.L.Cardy, Phys. Rev. Lett. 54 (1985) 1354

[20] P.Christe and F.Ravanini, NORDITA preprint 88/17P

[21] A.Cappelli, Phys. Lett. 185B (1987) 82

[22] I.K.Kostov, SPhT/88-035

[23] F.Ravanini, Mod. Phys. Lett. A3 (1988) 271 


\section{Appendix A:}

Proof of the equivalence between our characters (3.18) and those obtained by using GKO construction [13] on the coset:

$$
\frac{S U^{k}(2) \times S U^{1}(2) \times S U^{1}(2)}{S U^{k+2}(2)}
$$

The spin $l$ character of $S U^{N}(2)$ is

$$
\chi_{N, l}(\theta, q)=\prod_{n=1}^{\infty}\left(1-q^{n}\right)^{-1}\left(1-q^{n} e^{i \theta}\right)^{-1}\left(1-q^{n} e^{-i \theta}\right)^{-1} \Delta_{N, l}(\theta, q)
$$

with:

$$
\Delta_{N, l}(\theta, q)=q^{\frac{l(l+1)}{\lambda}} \sum_{n \in \mathbf{Z}} q^{\lambda n^{2}+(2 l+1) n}\left[e^{i(\lambda n+l) \theta}-e^{-i(\lambda n+l) \theta}\right]
$$

and: $\lambda=N+2$. In particular:

$$
\chi_{1, \epsilon}(\theta, q)=\prod_{n=1}^{\infty}\left(1-q^{n}\right)^{-1} \sum_{m \in \mathbf{Z}+\epsilon} q^{m^{2}} e^{i m \theta}
$$

with $\epsilon=0, \frac{1}{2}$. We want to show that:

$$
\chi_{N, l}(\theta, q) \chi_{1, \epsilon}(\theta, q) \chi_{1, \epsilon}(\theta, q)=\sum_{l^{\prime}} \chi_{N+2, l^{\prime}}(\theta, q) \chi_{l^{\prime}}(q)
$$

where $\chi_{l}(q)$ are our characters (3.18).

Denominators match, let us examine the numerators. The left hand side of (A.3) is:

$$
\left[\sum_{m_{1} \in \mathbf{Z}+\epsilon_{1}} q^{m_{1}^{2}} e^{i m_{1} \theta}\right]\left[\sum_{m_{2} \in \mathbf{Z}+\epsilon_{2}} q^{m_{2}^{2}} e^{i m_{2} \theta}\right]\left\{q^{\frac{l(l+1)}{\lambda}} \sum_{n \in \mathbf{Z}} q^{\lambda n^{2}+(2 l+1) n}\left[e^{i(\lambda n+l) \theta}-e^{-i(\lambda n+l+1) \theta}\right]\right\}
$$

Let us set:

$$
\begin{aligned}
m_{1} & =n+m_{1}^{\prime} \\
m_{2} & =n+m_{2}^{\prime} \\
l^{\prime} & =l+m_{1}^{\prime}+m_{2}^{\prime}
\end{aligned}
$$

we can thus write (A.4) as:

$$
\sum_{m_{1}^{\prime} \in \mathbf{Z}+\epsilon_{1}} \sum_{m_{2}^{\prime} \in \mathbf{Z}+\epsilon_{2}} q^{A} q^{\frac{l^{\prime}\left(l^{\prime}+1\right)}{\lambda+2}} \sum_{n \in \mathbf{Z}} q^{(\lambda+2) n^{2}+\left(2 l^{\prime}+1\right) n}\left[e^{i\left((\lambda+2) n+l^{\prime}\right) \theta}-e^{-i\left((\lambda+2) n+l^{\prime}+1\right) \theta}\right]
$$




$$
=\sum_{m_{1}^{\prime} \in \mathbf{Z}+\epsilon_{1}} \sum_{m_{2}^{\prime} \in \mathbf{Z}+\epsilon_{2}} q^{A} \chi_{N+2, l^{\prime}}(\theta, q)
$$

with:

$$
A=\frac{l(l+1)}{\lambda}+m_{1}^{2 \prime}+m_{2}^{2 \prime}-\frac{l^{\prime}\left(l^{\prime}+1\right)}{\lambda+2}
$$

Let us set:

$$
\begin{aligned}
& m_{1}^{\prime}+m_{2}^{\prime}=(\lambda+2) M+\left(l^{\prime \prime}-l\right) \\
& m_{1}^{\prime}-m_{2}^{\prime}=K
\end{aligned}
$$

with $M \in \mathbf{Z}, K \in \mathbf{Z}+\frac{1}{2}$ and $l^{\prime \prime}-l=1, \cdots, \lambda+2$ and the condition that if $K$ is half integer also $\left(l^{\prime \prime}-l\right)$ is such and if $K$ is even also $\left(l^{\prime \prime}-l\right)$ is such. By using the symmetry:

$$
\chi_{N, l+\lambda}(\theta, q)=\chi_{N, \lambda}(\theta, q)
$$

we can decouple the sum over $M$ then with simple manipulations we can write:

$$
A=\frac{\left[l^{\prime \prime} \lambda-l(\lambda+2)-1\right]^{2}-1}{2 \lambda(\lambda+2)}+\frac{K^{2}}{2}
$$

and we obtain:

$$
=\sum_{M \in \mathbf{Z}} \sum_{l^{\prime \prime}=1}^{\lambda+2} q^{\frac{[M \lambda(\lambda+2)+x]^{2}-1}{2 \lambda(\lambda+2)}} \chi_{N+2, l^{\prime \prime}}(\theta, q) \sum_{K \in \mathbf{Z}+\epsilon} q^{\frac{K^{2}}{2}}
$$

finally by using the symmetry

$$
\chi_{N,-l-1}(\theta, q)=-\chi_{N, l}(\theta, q)
$$

we end up with:

$$
=\sum_{l} \chi_{N+2, l}(\theta, q) \chi_{l}(q)
$$

with:

$$
\chi_{l}(q)=\frac{1}{\eta^{2}} \sum_{M \in \mathbf{Z}} \sum_{K \in \mathbf{Z}+\epsilon}\left\{q^{\frac{[M \lambda(\lambda+2)+x]^{2}-1}{2 \lambda(\lambda+2)}}-q^{\frac{[M \lambda(\lambda+2)+\tilde{x}]^{2}-1}{2 \lambda(\lambda+2)}}\right\}
$$

where $\tilde{x}=-l^{\prime \prime} \lambda-l(\lambda+2)-(\lambda+1)$.

These can be recognized as our characters (3.18) by setting $\lambda=2 n$, noting that using $\Gamma^{\prime}$ we can write $\tilde{x}$ as $(2 n+1) x$ if $l^{\prime \prime}-l$ is integer and $\tilde{x}$ as $(2 n+1) x+2 n(n+1)$ if $l^{\prime \prime}-l$ is half integer. Since in this last case $K$ must be half integer these are exactly the D fields of eq.(3.18) while if $l^{\prime \prime}-l$ is integer we have two possibilities:

i] $l$ and $l^{\prime \prime}$ separately half integers, then $x$ is even and we have A and C fields of eq.(3.18)

i] $l$ and $l^{\prime \prime}$ separately integers, then $x$ is odd and we have the B fields of eq.(3.18) 


\section{Appendix B:}

Description of our characters (3.18) as product of Supervirasoro and Ising characters.

The characters of the Supersymmetric models are [13,21]:

Neveu-Schwarz:

$$
\chi_{N S, \lambda}(\tau)=\sum_{n}\left\{q^{\frac{(N n+\lambda)^{2}}{4 N}}-q^{\frac{(N n+\tilde{\lambda})^{2}}{4 N}}\right\} \prod_{n=1}^{\infty} \frac{1+q^{n-\frac{1}{2}}}{1-q^{n}}
$$

where $\lambda$ must be even and $N=2 m(m+2)$ ( $m$ is the level of the theory); $\lambda=r(m+2)-m s$ $\tilde{\lambda}=r(m+2)+m s$

$$
\chi_{N{ }_{N, \lambda}}(\tau)=\chi_{N S, \lambda}(\tau+1),
$$

Ramond:

$$
\chi_{R, \lambda}(\tau)=\sum_{n}\left\{q^{\frac{(N n+\lambda)^{2}}{4 N}}-q^{\frac{(N n+\tilde{\lambda})^{2}}{4 N}}\right\} \prod_{n=1}^{\infty} \frac{1+q^{n}}{1-q^{n}}
$$

For the Ising model we have:

$$
\begin{gathered}
\chi_{0}+\chi_{\epsilon}=d_{\frac{1}{2}, \frac{1}{2}}=\prod_{n=1}^{\infty}\left(1+q^{n-\frac{1}{2}}\right) \\
\chi_{0}-\chi_{\epsilon}=d_{0, \frac{1}{2}}=\prod_{n=1}^{\infty}\left(1-q^{n-\frac{1}{2}}\right) \\
\chi_{\sigma}=d_{\frac{1}{2}, 0}=q^{\frac{1}{16}} \prod_{n=1}^{\infty}\left(1+q^{n}\right)
\end{gathered}
$$

Let us take $m$ even ( $m=$ level of the Supersymmetric model), say $m=2 k$. Then $N=2 m(m+2)=8 k(k+1)$ and

$$
\lambda=r(m+2)-s m=2(r(k+1)-s k) \equiv 2 \mu
$$

Let us call $\chi_{A}(x), \chi_{B}(x), \chi_{C}(x)$ the characters of the fields of type A,B,C ; let us separe the $\mathrm{D}$ fields in $D_{1}$ and $D_{-1}$ depending on the value of the bottom component and let us introduce the following shorthand notation:

$$
\left(\begin{array}{l}
x \\
z
\end{array}\right)=\frac{1}{\eta^{2}} \sum_{l_{1}, l_{2}} q \frac{\left[l_{1} 4 n(n+1)+x\right]^{2}-1}{8 n(n+1)}+\frac{\left[4 l_{2}+z\right]^{2}}{8}
$$

then we have:

$$
\chi_{A}(\mu)=\left(\begin{array}{c}
\mu \\
0
\end{array}\right)-\left(\begin{array}{c}
\tilde{\mu} \\
0
\end{array}\right) \quad \mu \quad \text { even }
$$




$$
\begin{gathered}
\chi_{B}(\mu)=\left(\begin{array}{c}
\mu \\
0
\end{array}\right)-\left(\begin{array}{c}
\tilde{\mu} \\
2
\end{array}\right) \quad \mu \quad \text { odd } \\
\chi_{C}(\mu)=\left(\begin{array}{c}
\mu \\
2
\end{array}\right)-\left(\begin{array}{c}
\tilde{\mu} \\
2
\end{array}\right) \quad \mu \quad \text { even } \\
\chi_{D_{1}}(\mu)=\left(\begin{array}{c}
\mu \\
1
\end{array}\right)-\left(\begin{array}{c}
\tilde{\mu} \\
1
\end{array}\right) \\
\chi_{D_{-1}}(\mu)=\left(\begin{array}{c}
\mu \\
-1
\end{array}\right)-\left(\begin{array}{c}
\tilde{\mu} \\
-1
\end{array}\right)
\end{gathered}
$$

where $\tilde{\mu}=\mu(2 k+1)$ if the field is of type A,B or $\mathrm{C}$ and $\tilde{\mu}=\mu(2 k+1)+2 k(k+1)$ if the field is of type D. Notice that these are the same relations which appear in the NS and $\mathrm{R}$ characters respectively for the supersymmetric model.

One can show by direct multiplication that if $\mu$ is even:

$$
\begin{aligned}
& \chi_{A}(\mu)+\chi_{C}(\mu)=\chi_{N S, 2 \mu}\left(\chi_{0}+\chi_{\epsilon}\right) \\
& \chi_{A}(\mu)-\chi_{C}(\mu)=\chi_{\tilde{N} S, 2 \mu}\left(\chi_{0}-\chi_{\epsilon}\right)
\end{aligned}
$$

if $\mu$ is odd:

$$
\chi_{B}(\mu)-\chi_{B}(\tilde{\mu})=\chi_{N S, 2 \mu}\left(\chi_{0}+\chi_{\epsilon}\right)
$$

and for the Ramond sector:

$$
\chi_{D_{1}}(\mu)-\chi_{D_{-1}}(\mu)=\chi_{R, 2 \mu} \chi_{\sigma}
$$

\section{Appendix C:}

C1] Equivalence between our characters (3.25) for the $\mathrm{c}=4 / 5$ model and the known ones $[11,20,22]$.

The $\mathrm{c}=4 / 5$ model is the $\mathrm{p}=4$ case of the Fateev-Zamolodchikov (F-Z) series [11]. The general expression of the characters of these models is the following:

$$
\begin{aligned}
\chi\left(\begin{array}{ll}
n_{1} & m_{1} \\
n_{2} & m_{2}
\end{array}\right)= & \\
\sum_{g \in G} \operatorname{sign}(g) \sum_{l_{1}, l_{2} \in \mathbf{Z}} q & \left\{\frac{\left[(p+1)\left(n_{1}+n_{2}\right)-p\left(m_{1}+m_{2}\right)+\left(l_{1}+l_{2}\right) p(p+1)\right]^{2}-4}{4 p(p+1)}\right. \\
& \left.+\frac{\left[(p+1)\left(n_{1}-n_{2}\right)-p\left(m_{1}-m_{2}\right)+3\left(l_{1}-l_{2}\right) p(p+1)\right]^{2}}{12 p(p+1)}\right\}
\end{aligned}
$$


where G is the "Weyl" group of $\mathrm{SU}(3)$ which is generated by $[20,22] \mathbf{- 1}$ and $g_{1}, g_{2}$ defined as:

$$
\begin{gathered}
g_{1}=\left\{\begin{array}{l}
n_{1} \rightarrow n_{2} \\
n_{2} \rightarrow-n_{1}-n_{2}
\end{array}\right\} \\
g_{2}=\left\{\begin{array}{l}
m_{1} \rightarrow m_{2} \\
m_{2} \rightarrow-m_{1}-m_{2}
\end{array}\right\}
\end{gathered}
$$

We give the proof in two steps: first one can see that the lattices sums are the same. Setting $\mathrm{p}=4$ in (C.1) we have:

$$
\sum_{l_{1}, l_{2} \in \mathbf{Z}} q^{\left\{\frac{\left[x+20\left(l_{1}+l_{2}\right)\right]^{2}-4}{80}+\frac{\left[z+60\left(l_{1}-l_{2}\right)\right]^{2}}{240}\right\}}
$$

provided we set:

$$
\begin{aligned}
& x=5\left(n_{1}+n_{2}\right)-4\left(m_{1}+m_{2}\right) \\
& z=5\left(n_{1}-n_{2}\right)-4\left(m_{1}-m_{2}\right)
\end{aligned}
$$

and this is exactly the lattice sum of eqs.(3.25).

Second: one can see directly, by inspection, that the two "Weyl" groups are the same.

We give for completeness the representatives of the F-Z corrispondent of our fields:

$$
\begin{gathered}
I=\left(\begin{array}{ll}
1 & 1 \\
1 & 1
\end{array}\right) \\
\epsilon=\left(\begin{array}{ll}
1 & 2 \\
1 & 2
\end{array}\right) \\
\sigma=\left(\begin{array}{ll}
1 & 1 \\
1 & 2
\end{array}\right)+\left(\begin{array}{ll}
1 & 2 \\
1 & 1
\end{array}\right) \\
\psi=\left(\begin{array}{ll}
1 & 1 \\
1 & 3
\end{array}\right)+\left(\begin{array}{ll}
1 & 3 \\
1 & 1
\end{array}\right)
\end{gathered}
$$

C2] In the case of the $c=10 / 7$ model the proof is similar to the previous one. This time $\mathrm{p}=6$, and in our model only a subset of the fields of the F-Z model is present, namely those with $\left(n_{1}-n_{2}\right)=0 \bmod 3$. The only non trivial point is that in order to match between the two lattice sums we have to sum on different F-Z fields obtained acting with the group generated by:

$$
g_{3}=\left\{\begin{array}{l}
n_{1} \rightarrow n_{2} \\
n_{2} \rightarrow p-n_{1}-n_{2}
\end{array}\right\}
$$


indeed this is typical of the models of the complementary series in the cases in which $\mathrm{p}$ is multiple of 3 [20]. in this way we can write F-Z lattice sum as:

$$
\sum_{l_{1}, l_{2} \in \mathbf{Z}} q^{\left\{\frac{\left[x+42\left(l_{1}+l_{2}\right)\right]^{2}-4}{168}+\frac{3\left[z+14\left(l_{1}-l_{2}\right)\right]^{2}}{168}\right\}}
$$

provided we set:

$$
\begin{aligned}
& x=7\left(n_{1}+n_{2}\right)-6\left(m_{1}+m_{2}\right) \\
& z=\frac{7}{3}\left(n_{1}-n_{2}\right)-2\left(m_{1}-m_{2}\right)
\end{aligned}
$$

which is exactly our lattice sum. At this point it is only matter of a long but straightforward check to identify the two "Weyl" groups so completing the proof. For sake of completness we report here the F-Z correspondent of our fields

$$
\begin{gathered}
I=\left(\begin{array}{ll}
1 & 1 \\
1 & 1
\end{array}\right) \\
A=\left(\begin{array}{ll}
2 & 1 \\
2 & 2
\end{array}\right)+\left(\begin{array}{ll}
2 & 2 \\
2 & 1
\end{array}\right) \\
B=\left(\begin{array}{ll}
2 & 2 \\
2 & 2
\end{array}\right) \\
C=\left(\begin{array}{ll}
2 & 3 \\
2 & 3
\end{array}\right) \\
D=\left(\begin{array}{ll}
2 & 1 \\
2 & 1
\end{array}\right) \\
E=\left(\begin{array}{ll}
1 & 1 \\
1 & 2
\end{array}\right)+\left(\begin{array}{ll}
1 & 2 \\
1 & 1
\end{array}\right) \\
F=\left(\begin{array}{ll}
1 & 2 \\
1 & 2
\end{array}\right) \\
G=\left(\begin{array}{ll}
1 & 3 \\
1 & 3
\end{array}\right)
\end{gathered}
$$

Sum over $g_{3}$ is understood. 


\section{Appendix D:}

In this appendix we shall derive certain identities that are used in the computation of correlation functions. Consider the integral:

$$
N\left(a, b, a^{\prime}, b^{\prime}\right) \equiv \int_{0}^{1} d x \int_{0}^{1} d y I(x, y)
$$

where:

$$
I(x, y)=x^{a}(1-x)^{b} y^{a^{\prime}}(1-y)^{b^{\prime}}(x-y)^{-2}
$$

and

$$
\frac{a}{a^{\prime}}=\frac{b}{b^{\prime}}=-\rho
$$

we have the following identities:

$$
\begin{aligned}
& \int_{0}^{1} d x \int_{0}^{1} d y(x-y) I(x, y)\left[\frac{a}{x}-\frac{b}{1-x}-\frac{1}{x-y}\right]=0 \\
& \int_{0}^{1} d x \int_{0}^{1} d y(x-y) I(x, y)\left[\frac{a^{\prime}}{x}-\frac{b^{\prime}}{1-x}-\frac{1}{x-y}\right]=0
\end{aligned}
$$

where we have used the fact that they are total derivatives with respect to $x$ and $y$ respectively and have assumed that the end point do not contribute. Multiplying (D.3) by $\rho$ and adding to eq.(D.2) we obtain (suppressing the argument of $N$ )

$$
\begin{aligned}
N & =\frac{1}{\rho-1} \iint d x d y x^{a}(1-x)^{b} y^{a^{\prime}}(1-y)^{b^{\prime}}\left[\frac{a}{x y}+\frac{b}{(1-x)(1-y)}\right] \\
& =\frac{1}{\rho-1}\left[\frac{a \Gamma(a) \Gamma(b+1) \Gamma\left(a^{\prime}\right) \Gamma\left(b^{\prime}+1\right)+b \Gamma(a+1) \Gamma(b) \Gamma\left(a^{\prime}+1\right) \Gamma\left(b^{\prime}\right)}{\Gamma(a+b+1) \Gamma\left(a^{\prime}+b^{\prime}+1\right)}\right. \\
& =\frac{\rho^{2}}{\rho-1} \frac{1}{a b(a+b)}\left[\frac{\Gamma(a+1) \Gamma(b+1) \Gamma\left(a^{\prime}+1\right) \Gamma\left(b^{\prime}+1\right)}{\Gamma(a+b) \Gamma\left(a^{\prime}+b^{\prime}\right)}\right.
\end{aligned}
$$

This is exactly the expression found in the appendix A of [9b]. Now let us consider the moments:

$$
<a x+a^{\prime} y>\equiv \frac{1}{N} \int_{0}^{1} d x \int_{0}^{1} d y I(x, y)\left(a x+a^{\prime} y\right)
$$

Note that $\langle x\rangle$ and $\langle y\rangle$ separately would depend on the ordering of contours but the above combination does not as is evident by taking the residue which turns out to be a total derivative. We have the following identities:

$$
\begin{aligned}
& \int_{0}^{1} d x \int_{0}^{1} d y\left[\frac{a+1}{x}-\frac{b+1}{1-x}-\frac{2}{x-y}\right] x(1-x) I(x, y)=0 \\
& \int_{0}^{1} d x \int_{0}^{1} d y\left[\frac{a^{\prime}+1}{y}-\frac{b^{\prime}+1}{1-y}+\frac{2}{x-y}\right] y(1-y) I(x, y)=0
\end{aligned}
$$


as they are total derivatives. Adding (D.6) and (D.7) we obtain:

$$
<a x+a^{\prime} y>=\left(a+a^{\prime}\right) \frac{a}{a+b}
$$

This is again consistent with the results of ref. [9b] for $\langle x\rangle$ and $\langle y\rangle$ however as pointed out above $\langle x\rangle$ and $\langle y\rangle$ are ambiguous. In fact one can show that choosing any particular ordering of contours $\langle x\rangle$ and $\langle y\rangle$ are given by eq.(B.3) of ref.[9b] plus some correction terms. However these correction terms drop out in the combination $\left.<a x+a^{\prime} y\right\rangle$. We emphasize that one must always use this combination in order to get unambiguous results.

Finally we shall also need the moments:

$$
<\frac{a}{x}+\frac{a^{\prime}}{y}>\equiv \frac{1}{N} \int_{0}^{1} d x \int_{0}^{1} d y I(x, y)\left(\frac{a}{x}+\frac{a^{\prime}}{y}\right)
$$

This combination is again unambiguous. We have the following identities:

$$
\begin{aligned}
& \int_{0}^{1} d x \int_{0}^{1} d y\left[\frac{a}{x}-\frac{b+1}{1-x}-\frac{2}{x-y}\right](1-x) I(x, y)=0 \\
& \int_{0}^{1} d x \int_{0}^{1} d y\left[\frac{a^{\prime}}{y}-\frac{b^{\prime}+1}{1-y}+\frac{2}{x-y}\right](1-y) I(x, y)=0
\end{aligned}
$$

Adding (D.10) and (D.11) we obtain:

$$
<\frac{a}{x}+\frac{a^{\prime}}{y}>=(1-\rho)(a+b)
$$

\section{Figure Captions}

Fig.1 Parallelogram representation of the torus in the limit $\tau \rightarrow i \infty$

Fig.2 The contours in the s-channel

Fig.3 The contours in the t-channel 